\title{
Die Bundestagswahl 2013: Demokratische Beteiligungslücke und Dauerkrise der SPD
}

Jörg Michael Dostal*

\section{Inhaltsverzeichnis}

I. Einleitung

II. Analyse der Bundestagswahl 2013

III. Theoretische Ansätze: Krise der Demokratie, Erosion der Volksparteien und demokratische Beteiligungslücke

IV. Die Dauerkrise der SPD

V. Fazit

\section{$<$ Abstract $>$}

Obwohl die CDU/CSU bei der Bundestagswahl 2013 - hauptsächlich auf Kosten ihres ehemaligen Koalitionspartners FDP - einen relativ hohen Stimmenanteil erzielen konnte, ist das deutsche Parteiensystem in einer zweifachen Strukturkrise gefangen. Erstens hat die Wahlbeteiligung so stark abgenommen, dass ein zunehmender Bindungsverlust zwischen den Wählern und den beiden traditionellen Volksparteien (CDU/CSU und SPD) festgestellt werden kann, woraus sich eine fortdauernde Erosion beider Volksparteien ergibt. Zweitens handelt es sich hierbei um eine asymmetrische Krise, denn die SPD leidet unter einem dramatischen Stimmen- und Mitgliederverlust, während die CDU/CSU vergleichsweise moderatere Einbußen zu verzeichnen hat. Ein

\footnotetext{
* Associate Professor, Graduate School of Public Administration, Seoul National University
} 
Hauptgrund für diese Entwicklung besteht in der zunehmenden sozialen Segregation der bundesdeutschen Gesellschaft, die sich unter anderem in einer stark sinkenden Wahlbeteiligung benachteiligter sozialer Schichten niederschlägt, während bürgerliche Wähler weiterhin in höherer Zahl von ihrem Wahlrecht Gebrauch machen. Hieraus ergibt sich eine demokratische Beteiligungslücke, die vor allem die SPD und im Osten Deutschlands auch die Partei Die Linke trifft. Beide wären jedoch auf die Wahlunterstützung durch benachteiligte Schichten stärker angewiesen als die bürgerlichen Parteien.

Key Words: Bundestagswahl 2013, Deutschland, Sinkende Wahlbeteiligung, Sozialdemokratische Partei Deutschlands (SPD), Krise der Volksparteien

\section{Einleitung}

Die Bundestagswahl am 23. September 2013 fand in einem widersprüchlichen politischen Umfeld statt. Einerseits war sie bestimmt von Verunsicherung über die seit 2008 anhaltende globale Wirtschaftskrise und die Befürchtung vieler Deutscher, dass die gemeinsame Währung des Euroraums (der Euro) sich als labil erweisen könnte. Sorge bereitete auch, dass eine Stabilisierung des Euro hohe deutsche Transferzahlungen in eurozonenweite gemeinsame Haftungs- oder Rettungsfonds für Staats- und Bankenschulden erzwingen könnte. Darin sahen viele Deutsche eine Bedrohung für die Solidität der deutschen Staatsfinanzen. Außerdem befürchteten viele Wähler, dass hohe zukünftige deutsche Staatsausgaben für die "Rettung" des Euro oder die Mithaftung für Staatsschulden anderer Euroländer auf weitere staatliche Aufgaben "durchschlagen" könnten. Eine Meinungsumfrage ergab, dass Staatsverschuldungund Inflation die Sorgenliste der Deutschen anführten, dicht gefolgt von den Themen Arbeitslosigkeit, Bildung und Stabilität der Altersrenten (European Commission, 2013: T 28-29). 
Andererseits erschien den meisten Deutschen die relative Positionierung Deutschlands im Rahmen der Wirtschafts- und Eurokrise weniger bedrohlichals die vieler anderer Mitgliedsländer der Europäischen Union (European Commission, 2013: T 20-21).

Für Verunsicherung sorgte wiederum, dass finanzpolitisch entscheidende Fragen wie die Sanierung von angeschlagenen Banken durch den Steuerzahler und Szenarien für die Umschuldung notleidender südlicher Eurostaaten im deutschen Bundestag in einer Form verhandelt wurden, die für die Wähler schwer verständlich war. Klare Unterschiede zu diesen Themen waren zwischen den im Parlament vertretenen Parteien - vor allem in Bezug auf die Wahlkonkurrenz zwischen CDU/CSU und SPD - nicht erkennbar. Die Eurokrisenpolitik wurde darum für den Wahlkampf als zu kompliziert stillgelegt. Dies gab der CDU/CSU die Gelegenheit, das Thema Wirtschaft für sich zu monopolisieren und mit der behaupteten Führungskompetenz der Kanzlerin Angela Merkel zu verbinden.

Trotz der teilweisen Überlagerung der Themen des Wahlkampfs durch Banken- und Eurokrise ergaben sich jedoch auch Anknüpfungspunkte für die drei Oppositionsparteien SPD, Die Linke und Grüne. So sah eine weitere Meinungsumfrage im Wahljahr die Arbeitslosigkeit - trotz sinkender Tendenz - weiterhin als Hauptsorge der Deutschen, gefolgt von den Themen Inflation, wirtschaftliche Stabilität, Bildung und Sicherheit der Altersrenten (gfk-Verein, 2013). In der Beurteilung der Themenkompentenz der beiden Großparteien ergab sich in der Summe jedoch eine eindeutige Führungsposition der Union. Sie wurde von den Wählern als die Partei angesehen, die die Wirtschaft voranbringt (CDU/CSU 57 Prozent, SPD 21 Prozent), Arbeitsplätze garantiert (48 Prozent versus 26 Prozent) und die Euro und Schuldenkrise in den Griff bekommen könnte (42 Prozent versus 18 Prozent). Bei den sozialpolitischen Themen Familienpolitik, Altersversorgung und bezahlbaren Energiepreisen lagen CDU/CSU und SPD jeweils gleichauf. Die SPD erzielte nur im Bereich soziale Gerechtigkeit klar bessere Werte (40 Prozent SPD versus 26 Prozent CDU/CSU). Sie lag außerdem beim Thema Gesundheitspolitik leicht vor der Union (33 Prozent versus 26 Prozent) (Infratest Dimap, 2013a: 9-10).1)

1) Die Beurteilung der Parteienkompetenz ist maßgeblich durch die relative Popularität der Parteien 


\section{Analyse der Bundestagswahl 2013}

\section{Vergleichende Parteienanalyse}

Die Bundestagswahl 2013 brachte einen klaren Wahlsieg der CDU/CSU. Die SPD konnte sehr geringe Zugewinne erzielen und die kleineren Parteien, Linke und Grüne, verloren moderat. Die marktliberale FDP musste dagegen dramatische Verluste hinnehmen und ist zum ersten Mal in der Geschichte der Bundesrepublik nicht mehr im Bundestag vertreten. Die neu gegründete "Alternative für Deutschland" (AfD) erzielte mit 4,7 Prozent einen Achtungserfolg. Die Wahlergebnisse und die Wählerwanderungen zwischen den Parteien sind in Tabelle 1 zusammengefasst.

\section{$\mathrm{CDU} / \mathrm{CSU}$}

Die Wahlkampagne der CDU/CSU war wie schon im Jahr 2009 auf die Kanzlerin zugeschnitten. Sie hat durch ihre Rolle als Krisenmanagerin in der Eurokrise und durch die von ihr verantwortete programmatische Modernisierung der CDU weiter an Statur gewonnen. Wie schon 2009 war der Wahlkampf auf das politische Zentrum ausgerichtet. Unter Merkel ist die CDU weder neoliberal-rechts noch christlich-sozial. Vertreter dieser Flügel werden zwar in der Partei geduldet, bestimmen aber nicht ihr Bild. Wie schon 2009 folgte die Kanzlerin auch 2013 der Strategie der kalkulierten Demobilisierung, die einerseits auf programmatische Zuspitzungen verzichtet und andererseits alle kontroversen Themen wie z.B. den Mindestlohn oder die Details der angekündigten Energiewende meidet, um der Opposition keine Mobilisierungsmöglichkeiten zu geben (Blätte, 2010).

Die Union konnte ihr Wahlergebnis im Vergleich zu 2009 mit 41,5 Prozent (plus 7,7 Prozent) deutlich verbessern und den langfristigen Abwärtstrend der Partei in der

bestimmt, d.h. die deutlich geringere Wählerunterstützung der SPD im Vergleich zur Union erklärt zum Teil auch die niedrigere Kompetenzzuschreibung. 
Wählergunst umkehren. Allerdings war der Erfolg hauptsächlich auf die Kannibalisierung des Koalitionspartners FDP zurückzuführen, die für mehr als die Hälfte der durch die Union hinzugewonnen Stimmen verantwortlich war. Es handelte sich also um eine Umverteilung innerhalb des bürgerlichen Lagers. Weitere wichtige Ergebnisse waren die Rückgewinnung früherer Nichtwähler wie auch die Vermeidung größerer Stimmenverluste an die neugegründete eurokritische AfD. Direkte Nettostimmengewinne von den drei Oppositionsparteien gelangen der Union dagegen nur in sehr geringem Maß.2)

\section{SPD}

Die Sozialdemokraten erreichten mit 25,7 Prozent (plus 2,7 Prozent) ein leicht verbessertes Wahlergebnis im Vergleich zu ihrer Rekordniederlage 2009. Dennoch war dieses Resultat das zweitschlechteste in der bundesdeutschen Geschichte. Obwohl die SPD sich darum bemühte, die mit dem letzten SPD-Kanzler Gerhard Schröder verbundene Politik der sogenannten "Agenda 2010" und der "Hartz-Reformen" (d.h. die deregulierenden Arbeitsmarkt - und Finanzmarktreformen sowie die Steuersenkungen für Großverdiener) hinter sich zu lassen, und deutlich in Richtung sozialpolitischer Themen ausschwenkte, wurde sie dabei als wenig glaubwürdig wahrgenommen. Ihr Kanzlerkandidat Peer Steinbrück, ehemaliger Bundesfinanzminister der letzten Großen Koalition von CDU/CSU und SPD zwischen 2005 und 2009, war klar als Vertreter des marktorientierten Mehrheitsflügels der Partei profiliert. Die SPD-Wahlkampagne zu sozialen Themen passte daher nicht zum Kandidaten. Zusätzlich fiel Steinbrück durch verschiedene taktische Fehlgriffe wie z.B. Äußerungen zum niedrigen Gehalt des Bundeskanzlers ("Nahezu jeder Sparkassendirektor in Nordrhein-Westfalen verdient mehr als die Kanzlerin", FAZ, 29.12.2012) negativ auf. Bei weiblichen Wählern erreichte

2) Butzlaff (2013) weist aber zu Recht darauf hin, dass zwischen Union und SPD trotzdem eine überraschend große Stimmenwanderung in beide Richtungen stattfand. So verlor die Union gut 700,000 Stimmen an die SPD, die ihrerseits über 900,000 Stimmen an die Union abgab. Dieser Effekt bleibt bei einer Betrachtung der Nettostimmengewinne unsichtbar. 
er nur ausgesprochen niedrige Zustimmungswerte.3) Ein weiterer Kritikpunkt an Steinbrück war, dass die Entscheidung über seine Kanzlerkandidatur innerhalb der engeren Parteiführung und ohne Mitgliederentscheid erfolgte. Kritiker sahen dadurch eine Chance zur innerparteilichen Mobilisierung verspielt und beklagten einen "erbarmungswürdigen Zustand" der SPD (Walter, 2013).

Der SPD-Wahlkampf litt darunter, dass Steinbrück von den Wählern zu keinem Zeitpunkt als eine realistische Alternative zu Kanzlerin Merkel angesehen wurde. Außerdem wurde durch die Selbstfestlegung der SPD auf eine Wunschkoalition mit den Grünen sowie den Ausschluss einer Dreierkoalition mit der Partei Die Linke keine realistische Option für einen Regierungswechsel aufgezeigt. Diese rot-grüne Koalitionsoption war wegen der mangelnden Wählerunterstützung, die in allen Meinungsumfragen klar erkennbar war, nicht glaubwürdig: Die Wähler erwarteten vom Wahlausgang 2013 stattdessen mehrheitlich eine Große Koalition, in der die SPD die Rolle des Juniorpartners der CDU/CSU einnehmen würde. (Solche Erwartungen erwiesen sich nach den Wahlen auch als zutreffend: Union und SPD verständigten sich auf eine neue Große Koalition und Angela Merkel wurde am 17. Dezember mit den Stimmen der meisten Abgeordneten der CDU/CSU und der SPD erneut zur Bundeskanzlerin gewählt. Die SPD akzeptierte damit wie schon 2005 die Rolle des Juniorpartners der Union.)

\section{Die Linke}

Nach ihrem Rekordergebnis bei der Bundestagswahl 2009, bei der es der Partei Die Linke4) gelungen war, eine große Gruppe von enttäuschten SPD-Wählern zu sich

3) In der letzten Vorwahlumfrage der Forschungsgruppe Wahlen im September 2013 erzielte Angela Merkel eine Zustimmungsquote von 50 Prozent bei Männern und 58 Prozent bei Frauen, während Steinbrück von 40 Prozent der Männer aber nur von 28 Prozent der Frauen bevorzugt wurde. Eine Beobachterin merkte an, dass eine geschlechtsspezifische Lücke von 30 Prozentpunkten in der Unterstützung durch weibliche Wähler einen Wert darstellt, "auf [den] man in der Wahlforschung selten stößt" (Neu, 2013: 20). 
hinüberzuziehen, musste sie bei dieser Wahl Verluste hinnehmen und erreichte nur noch 8,6 Prozent der Stimmen (minus 3,3 Prozent). Besonders stark verlor die Partei in den fünf ostdeutschen Bundesländern an die neugegründete Alternative für Deutschland (AfD) (siehe unten). Ähnlich hohe Verluste ergaben sich auch in Richtung SPD und zu den Nichtwählern. Obwohl die Linke in vier der fünf neuen Bundesländer weiterhin zweitstärkste Partei nach der CDU ist, verlor sie alle 12 Direktmandate in den fünf ostdeutschen Bundesländern an die CDU (Bundeswahlleiter, 2013a: 3) und ist nun nur noch in vier Ostberliner Wahlbezirken stärkste Partei. In den alten Bundesländern waren die Verluste der Linken insgesamt sogar höher. Allerdings gelang der Partei hier unerwartet mit 5,2 Prozent der Wiedereinzug in den hessischen Landtag bei den dort gleichzeitig mit der Bundestagswahl stattfindenden Landtagswahlen. Als Probleme der Linken erwiesen sich die Themen Bankenrettung und Zukunft des Euro. Obwohl die Linke sich als klare Kritikerin der Bankenrettung aus Steuergeldern präsentierte, verlor sie Stimmen an die zu diesem Thema scheinbar radikaler argumentierende AfD. Die Linke hatte sich im Wahlkampf außerdem klar zur weiteren Existenz des Euro bekannt und damit innerparteiliche Kritiker der Eurowährung kaltgestellt. Es blieb unklar, ob die Unterstützung der Eurowährung und die Kritik an einem "neoliberalen Europa" wirklich zueinander passen.

Unabhängig vom wahlpolitischen Diskurs des Jahres 2013 erklären aber auch strukturelle Veränderungen im Profil der Partei den Stimmenverlust. Nach dem Ende der DDR war die Vorläuferorganisation der Linken, die PDS, in erster Linie ein

4) Die Partei "Die Linke" entstand aus der Vereinigung der ostdeutsch geprägten "Partei des demokratischen Sozialismus" (PDS) mit der hauptsächlich westdeutsch geprägten "Wahlalternative Arbeit und Soziale Gerechtigkeit"(WASG). Die WASG war eine Organisation von ehemaligen SPD - Mitgliedern und Gewerkschaftern, welche die SPD aus Protest gegen die von SPD-Kanzler Schröder durchgeführten marktliberalen Reformen und Sozialkürzungen ("Agenda 2010" und "Hartz -Reformen") verlassen hatten. Am 17. Juli 2005 benannte sich die PDS in Vorbereitung auf die Vereinigung mit der WASG in "Die Linkspartei" um und führte ein neues Parteilogo ein, das sie als "Die Linkspartei.PDS" auswies. Nach der endgültigen Vereinigung mit der WASG am 16. Juni 2007 wurde der Parteiname schließlich in "Die Linke" geändert. Allerdings wird die Partei weiterhin häufig als "Linkspartei" bezeichnet. 
Sammelbecken der durch die Vereinigung sozial und kulturell deklassierten Führungselite der DDR und besonders der mittleren und höheren Leitungskader der ostdeutschen Wirtschaft und Gesellschaft (Niedermayer, 2009: 99). Dieses Milieu verliert aber an Bedeutung und sorgt für eine Abschwächung der Verankerung der Linken in Ostdeutschland. Allerdings gelang der PDS seit den Hartz-Reformen der rot-grünen Schröder-Fischer-Regierung zwischen 2003 und 2005 zum ersten Mal die breite Mobilisierung von sozial schwachen Wählern und Arbeitslosen, die in der Vergangenheit eher SPD oder auch die Union wählten. Diese Wählergruppen sind bei Wahlen jedoch deutlich schwerer zu mobilisieren als sozial besser gestellte Wähler. So gesehen hat die Linke also spezifische Mobilisierungsprobleme bei der Wählergruppe der "Sozialstaatsenttäuschten" von der SPD geerbt. Es ist ihr nicht gelungen, die sozial schwachen Wechselwähler dauerhaft an sich zu binden.

\section{Grüne}

Die Grünen erreichten mit 8,4 Prozent (minus 2,3 Prozent) ein Ergebnis, das unter den Erwartungen lag. Nach der japanischen Atomkatastrophe in Fukushima waren die Grünen als führende Anti-Atompartei in Meinungsumfragen mehrmals als "neue Volkspartei" mit mehr als 20 Prozent Wählerzustimmung - und im Herbst 2010 und Frühjahr 2011 in einigen Meinungsumfragen sogar vor der SPD - taxiert worden. Diese hohen Erwartungen sollten sich für die Grünen jedoch nicht bestätigen. Es zeigte sich, dass die Merkel-CDU durch ihren nach Fukushima durchgeführten Richtungswechsel in der Atomfrage ein für die Grünen wichtiges Mobilisierungsthema entschärfen konnte. Die Welle der Unterstützung für die Grünen ebbte darum schnell wieder ab. Aus soziologischer Sicht erscheint dies auch nicht erstaunlich. Die Grünen haben sich zunehmend zu einer Partei neu-bürgerlicher Saturiertheit entwickelt. Das grüne Wählerklientel gehört überwiegend zu den Besserverdienern und ist im Gegensatz zur Wählerschaft der historischen Volksparteien (d.h. der CDU/CSU und der SPD) überdurchschnittlich akademisch gebildet. Dieses gesellschaftliche Milieu hat zwar 
expandiert, ist jedoch für die Begründung einer Volkspartei zahlenmäßig zu klein.

Zusätzlich zeigt sich das grün-bürgerliche Milieu auch intern gespalten. Obwohl die Grünen auf der Achse zwischen libertären und autoritären Werten klar libertär verortet sind, ist ihre politische Position auf einer Achse zwischen Marktliberalismus und sozialer Umverteilung weniger klar. Insofern bleibt fraglich, ob Grünwähler Steuererhöhungen tatsächlich unterstützen, die sie selbst treffen würden. Im Wahlprogramm der Grünen wurden jedoch z.B. Steuererhöhungen für Jahreseinkommen über 60,000 Euro und eine "grüne Vermögensabgabe" für Euro-Millionäre sowie Betriebsvermögen vorgeschlagen (Bündnis 90/Die Grünen, 2013: 82-83). Beobachter verorteten die Wahlkampfstrategie der Grünen u.a. deshalb als zu nahe an der Linkspartei. Statt sich auf Umweltfragen zu konzentrieren, für die den Grünen die Meinungsführerschaft zugeschrieben wird, versuchte die Partei, die soziale Frage zu besetzen. Kritiker schlussfolgerten daraus, dass "die Grünen (...) zwischen den linken Umverteilungsapologeten und den liberalen Umverteilungsfeinden zerrieben [wurden]" (Lühmann, 2013). Zusammenfassend lässt sich daher sagen, dass den Grünen die zahlenmäßige Begrenztheit und interne Gespaltenheit ihrer Wählerklientel zu schaffen machte.

\section{FDP}

Die FDP stürzte in der Wählergunst dramatisch ab und erreichte mit 4,8 Prozent (minus 9,8 Prozent) nicht mehr die für den Wiedereinzug in den Bundestag notwendige Stimmenzahl. Der Grund für dieses Wahldesaster lag darin, dass Wähler der CDU/CSU diesmal nicht bereit waren, die FDP aus wahltaktischen Gründen zu unterstützen. Die FDP hat eine lange Geschichte als Partei der sogenannten "Leihstimmen": CDU/ CSU-Wähler waren in der Vergangenheit häufig gewillt, durch die Wahl der FDP eine bürgerliche Mehrheit im Parlament herzustellen. Bei der Bundestagswahl 2009 erhielt die FDP noch außergewöhnlich viele solcher Leihstimmen von Wählern der CDU/CSU und erreichte dadurch das beste Ergebnis der Parteigeschichte (Kulick, 2011: 
205-207). Diesmal suggerierten die meisten Meinungsumfragen, dass die FDP die Fünfprozenthürde nicht nehmen würde. Darum sahen CDU/CSU-Wähler eine Leihstimme für die FDP als eine möglicherweise verlorene Stimme an und lehnten die "Mitleidsmasche" der FDP bei dieser Bundestagswahl ab (Sirleschtov, 2013).

Ursächlich für den Niedergang der FDP sind aber auch strukturelle Gründe. Historisch verstand sich die FDP noch nie als Volkspartei; sie wurde stattdessen als Interessenpartei der Arbeitgeber und als neoliberale Richtungspartei wahrgenommen. Zusätzlich wirkte sie auch als Lobby für Besserverdiener und erklärte sich selbst zur "Steuersenkungspartei". Obwohl die FDP in der Vergangenheit für sich in Anspruch nahm, auch sozialliberale Ideen und Bürgerrechte zu vertreten, sind diese Themen schon seit vielen Jahren im Profil nicht mehr sichtbar. Diese programmatische Verengung der FDP auf Wirtschaftsthemen erwies sich nun als fatal, da sie sich in der Koalition mit der CDU/CSU seit 2009 kaum profilieren konnte. Die Partei litt unter Auszehrung: Das Führungspersonal war unbeliebt, die Mitgliederzahlen fielen seit Jahren in noch höherem Tempo als bei den anderen Parteien und eine Verankerung in Ostdeutschland war nicht vorhanden. Zuletzt wurde der Partei auch im Bereich der Wirtschaftskompetenz nur noch von 4 Prozent der Wähler die führende Rolle zugesprochen, während der Koalitionspartner CDU/CSU einen Wert von 57 Prozent erreichte (Infratest Dimap, 2013a: 9). Ohne den Niedergang der FDP wäre der Wahlerfolg der CDU/CSU bei dieser Bundestagswahl deutlich bescheidener ausgefallen.

\section{Alternative für Deutschland (AfD)}

Die eurokritische AfD erreichte bei ihrer ersten Bundestagskandidatur aus dem Stand 4,7 Prozent der Stimmen und verfehlte den Einzug in den Bundestag nur sehr knapp. Die neue Organisation befindet sich gegenwärtig im Stadium einer patchwork-Partei, die zu einem single issue, nämlich der Eurokrise und der vermuteten finanziellen Haftung Deutschlands und/oder der deutschen Sparer und Steuerzahler, mobilisiert hat. Ähnlich wie in der Parteigründungsphase der Partei "Die Grünen" Anfang der achtziger Jahre 
sammeln sich in der AfD enttäuschte Wählergruppen, die auf den ersten Blick nicht zusammenpassen. Die Führung der neuen Partei besteht aus Professoren der Volkswirtschaftslehre, welche die Rettung von "systemrelevanten" Banken durch die Steuerzahler unter Führung der Merkel-CDU - und die darauf folgende Finanzkrise der öffentlichen Hand - kritisieren. Zusätzlich konnte die AfD einzelne Vertreter des enttäuschten konservativen Flügels der CDU für sich gewinnen. Sie verlangt Marktbereinigung und Abschreibung von Verlusten statt langfristiger Umschuldung und EU-weitem Krisenmanagement, nach dem Motto: "Lieber ein Ende mit Schrecken als ein Schrecken ohne Ende!" Sie möchte den Euro entweder wieder abschaffen oder eine Währungsgruppe der wirtschaftlich starken Euroländer unter Ausschluss der krisengeschüttelten Südländer (eine Art Euro der zwei Geschwindigkeiten) etablieren.

Obwohl die neue Partei erklärt, dass sie weder rechts noch links ist, kann man ihr wirtschaftspolitisches Profil als marktradikal bezeichnen. Soziale Forderungen werden von der AfD nicht erhoben. Ihre Wähler sind dagegen sozial sehr gemischt und häufig aus eher benachteiligten Schichten. Hochburgen der AfD liegen überwiegend in Ostdeutschland und die Wählerwanderung zur AfD erfolgte aus unterschiedlichen Lagern. Obwohl ehemalige FDP-Wähler wegen ihrer marktliberalen Haltung erwartungsgemäß die größte Gruppe stellten, folgen direkt dahinter ehemalige Wähler der Partei Die Linke. Frühere CDU/CSU-Wähler bildeten dagegen nur die drittgrößte Gruppe. Für die zukünftige Entwicklung der AfD werden zwei miteinander verbundene Punkte entscheidend sein. Erstens die weitere Entwicklung der Euro- und Finanzkrise und das damit zusammenhängende Mobilisierungspotential sowie zweitens die Frage, ob der AfD ein Vordringen in die Wählerschaft der CDU/CSU gelingt. Falls Merkel an sie gerichtete Erwartungen hinsichtlich der Euro- und Finanzkrise nicht erfüllen sollte, könnte sie schnell unter steigenden Druck seitens der AfD kommen, die dann möglicherweise ihrerseits (ähnlich wie die britische UK-Independence Party im Verhältnis zur dortigen Conservative Party) starken Druck auf das bürgerliche Lager ausüben könnte. 


\section{Strukturelle Veränderungen des politischen Systems: Sinkende Wahlbeteiligung und Dauerkrise der SPD}

Zusammenfassend lässt sich feststellen, dass die Parteikombination SPD-Die Grünen ("rot-grün") bei der Bundestagswahl 2013 in keiner einzigen der klassischen soziologischen Großgruppen eine Stimmenmehrheit gewinnen konnte (siehe Graphik 1). Die einzige Wählergruppe, bei der die SPD knapp vor der CDU/CSU liegt und in der eine klare Mehrheit links der Mitte besteht, sind die Arbeitslosen. Selbst hier wäre die SPD aber auf die Linke angewiesen, die bei den Arbeitslosen zusammen mit CDU/CSU und SPD die dritte Großpartei ist. Ein weiterer wichtiger Punkt ist, dass die beiden Hauptparteien CDU/CSU und SPD bei den Jungwählern weniger Zustimmung erfahren als bei der älteren Wählerschaft (siehe Graphik 2). Durch die generell höhere Wahlbeteiligung älterer Wähler profitieren insgesamt Union und SPD auf Kosten der kleineren Parteien, die hier weniger Schwankungen aufweisen.

Um die Analyse dieser Bundestagswahl abzurunden, müssen neben konjunkturellen Faktoren - dem Auf und Ab der Parteien von Wahl zu Wahl - auch die langfristig wirksamen Entwicklungstendenzen untersucht werden. Die Fragestellung lautet: Was ist eigentlich bemerkenswert am Wahlergebnis des Jahres 2013 in Bezug auf die strukturellen Veränderungen? Hier fallen vor allem zwei Resultate ins Auge, nämlich die geringe Wahlbeteiligung und die Dauerkrise der SPD. Aus struktureller Sicht ist die Wahlbeteiligung in Westdeutschland seit den siebziger Jahren und im vereinigten Deutschland seit 1990 - wie in fast allen anderen OECD-Demokratien - langsam über die Jahrzehnte zurückgegangen (Streeck und Schäfer 2013: 10-17). Zusätzlich war bei der Bundestagswahl 2009 ein regelrechter Einbruch der Wahlbeteiligung, nämlich ein Fall der Beteiligung von 77,7 Prozent bei der Wahl im Jahr 2005 - selbst bereits ein Negativrekord im Vergleich zu allen vorangegangenen Bundestagswahlen - auf 70,8 Prozent bei der Wahl 2009 festzustellen, der bei der Bundestagswahl 2013 mit 71,5 Prozent praktisch nicht umgekehrt wurde. Dieser Fall der bundesdeutschen Wahlbeteiligung auf historische Tiefststände wurde auch durch öffentliche Kampagnen, 
die für eine Wahlteilnahme im Jahr 2013 warben (Slogan: "Jede Stimme zählt!") und durch das Auftauchen einer neuen Flügelpartei in Gestalt der AfD, die eigentlich frühere Nichtwähler mobilisieren sollte, kaum beeinflusst.5)

Abgesehen vom allgemeinen Rückgang der Wahlbeteiligung bei Bundestagswahlen muss festgestellt werden, dass der SPD in diesem Zusammenhang wegen ihrer besonders drastischen Stimmenverluste des Jahres 2009 und der nur sehr schwachen Erholung des Jahres 2013 eine besondere Rolle zukommt. Die Krise der SPD ist viel dramatischer als die der CDU/CSU wie Tabelle 2 zeigt, in der das Mobilisierungspotential der Parteien bei den Bundestagswahlen im vereinigten Deutschland seit 1990 ausgewiesen wird.6) Die maximal erzielte Zahl der Zweitstimmen bei den Bundestagswahlen zwischen 1990 und 2013 wird mit 100 Prozent gezählt. Alle anderen Zweitstimmenergebnisse werden in Relation zur maximalen Stimmenmobilisierung gesetzt, um die relative Stärke der Parteien im Vergleich zu ihrem größten Wahlerfolg im vereinigten Deutschland anzuzeigen. Dabei zeigt sich, dass die Volatilität der Wählerunterstützung der SPD und die der kleineren Parteien deutlich höher ist als jene der CDU/CSU. Die besonders niedrige Wahlbeteiligung bei den Bundestagswahlen 2009 und 2013 ist deshalb vor allem ein SPD-Problem, d.h. der Absturz der Mobilisierungsfähigkeit der SPD erklärt auch zu einem Großteil die sinkende Wahlbeteiligung. Mehr als zwei Millionen der neuen Nichtwähler des Jahres 2009 kamen direkt von der SPD, während die CDU-Verluste an die Nichtwähler nur halb so hoch waren (Korte, 2011: 11; Tagesschau, 2009). Obwohl andere Parteien wie z.B. die FDP und die Linke ebenso eine sehr hohe Volatilität der Stimmen aufweisen, lassen sich diese häufiger als Stimmenwanderung (Wechsel von der FDP zur CDU

5) Tatsächlich "zählten" bei der Bundestagswahl 2013 weniger Stimmen als je zuvor für die Zusammensetzung des Bundestages, weil die Stimmenzahl der Parteien unterhalb der Fünfprozenthürde (FDP, AfD, Piraten und sonstige Parteien) mit 15,7 Prozent höher lag als bei allen früheren Bundestagswahlen im vereinigten Deutschland. Dies muss ebenfalls als Teil der Mobilisierungskrise von Union und SPD angesehen werden.

6) Bei der ersten gesamtdeutschen Bundestagswahl 1990 war das Parteiensystem noch nicht konsolidiert und Ost-Grüne und PDS erreichten nur aufgrund der für Ost- und Westdeutschland getrennten Fünfprozenthürden den Einzug in den Bundestag. 
2013 oder von der SPD zu den Linken 2009) statt als Wahlverweigerung erklären. Die Mobilisierungsprobleme der SPD werden im zweiten Teil dieses Artikels näher untersucht.

Zusammenfassend lässt sich sagen, dass die Bundestagswahl 2013 die Krise von CDU/CSU und SPD leicht entschärft hat, da beide Großparteien zum ersten Mal seit längerer Zeit wieder Stimmengewinne erzielen konnten, während der Block der kleineren Parteien im Parlament durch das Ausscheiden der FDP und die Verluste der Linken und Grünen geschrumpft ist. Trotzdem fand kein grundlegender Trendwechsel statt: Der Stimmenanteil der nicht im Parlament vertretenen Parteien erreichte ein Rekordniveau und der CDU/CSU-Erfolg war in erster Linie auf die Mobilisierungsstärke der Kanzlerin und die Schwäche der FDP zurückzuführen. Besonders bei jüngeren Wählern kann von einer Bewegung zurück zu den großen Parteien weiterhin keine Rede sein.

Der nächste Abschnitt stellt einige theoretische Perspektiven vor, die bei der Analyse der sinkenden Wahlbeteiligung und der anhaltenden Krise der SPD hilfreich sein können.

\section{Theoretische Ansätze: \\ Krise der Demokratie, Erosion der Volksparteien und Demokratische Beteiligungslücke}

In diesem Abschnitt werden drei theoretische Debatten, nämlich 1. die Diskussion einer Krise der Demokratie, 2. das Problem der Erosion der Volksparteien und 3. die demokratische Beteiligungslücke bei Wahlen aufgegriffen. Zunächst wird hinterfragt, ob es gerechtfertigt ist, von einer generellen Krise der Demokratie zu sprechen. Anschließend wird das Konzept der Volkspartei in Deutschland auf seine 
weitere Tragfähigkeit hin geprüft. Zuletzt folgt eine Analyse der wachsenden demokratischen Beteiligungslücke, die sich in Zeiten sinkender Wahlbeteiligung besonders bei den sozial schwächeren Wählergruppen geöffnet hat. Es wird untersucht, ob diese Beteiligungslücke die Wahlergebnisse in der Bundesrepublik direkt beeinflusst. Allerdings muss betont werden, dass diese Diskussion theoretischer Konzepte auf einige wenige Stimmen beschränkt ist, die hier hinsichtlich der Relevanz für die Analyse des wahlpolitischen Niedergangs der SPD ausgewählt wurden.

\section{Debatten über die Krise der Demokratie}

Die Diskussion einer Krise der Demokratie ist so alt wie die Verallgemeinerung demokratischer Ideen und Institutionen. Grundsätzlich wurden demokratische Prinzipien nach dem zweiten Weltkrieg auch in der Bundesrepublik mehrheitsfähig. Das Thema der Krise der Demokratie wurde danach vor allem in Folge der Studentenbewegung nach 1968 und in der Periode der sogenannten neuen sozialen Bewegungen (AntiAtomkraft-, Frauen-, Friedens-und Umweltbewegung) Anfang bis Mitte der achtziger Jahre thematisiert. In diese Periode fällt auch die Gründung der Grünen, die sich zunächst als "Anti-Parteien-Partei" und als Repräsentantin außerparlamentarischer Bewegungen im Parlament verstand. Das letzte Jahr der DDR zwischen der politischen Wende im Herbst 1989 und dem Ende im Herbst 1990 brachte eine eigene ostdeutsche Demokratisierungsbewegung hervor (z.B. die DDR-Allparteienregierung, die Bürgerbewegungen, die runden Tische zur Konfliktlösung und die Transformation der ostdeutschen Regierungs - in eine linke Oppositionspartei). Jedoch hinterlie $\beta$ dieser neue demokratische Impuls kaum Spuren im vereinigten Deutschland. Stattdessen hat sich das Thema einer Krise der Demokratie in der Bundesrepublik seit den neunziger Jahren wegen des schleichenden Erosionsprozesses der demokratischen Beteiligung zu einer dauerhaften, geradezu chronischen Debatte entwickelt.

Generell lässt sich die Diskussion der Krise der Demokratie aus zwei Richtungen führen. Einerseits betonen Autoren, die einer realistischen und institutionell- 
dezisionistischen Richtung anhängen, dass das demokratische politische System in erster Linie kohärenten repräsentativen Prinzipien folgen muss. Die Art und Weise, in der Entscheidungen getroffen werden sowie die Qualität der Entscheidungen selbst werden dagegen als zweitrangig angesehen. Als Krise der Demokratie wird darum eine Entscheidungsschwäche des politischen Systems angesehen. Diese Richtung sieht die Gefahr einer Krise der Demokratie vor allem wegen der möglichen Überlastung durch eine Vielzahl von Interessengruppen: Je mehr sich politische Führungsfiguren in langfristigen Prozessen um die Herstellung eines Konsenses bemühen, desto mehr lässt das Zutrauen in ihre Problemlösungsfähigkeit nach (Huntington, 1975: 78). Das Verlangen nach starker politischer Führung zur Überwindung einer "pluralistischen Stagnation" (der gegenseitigen Blockierung der Interessengruppen) war darum eines der zentralen Motive der Krisendiskussion in Demokratien der siebziger Jahre, taucht aber auch in der Gegenwart periodisch wieder auf.7) So werden Sorgen um die Entscheidungsfähigkeit demokratischer Systeme auch im Zusammenhang mit der ständig anwachsenden Komplexität fortgeschrittener Gesellschaften geäußert. Dabei wird betont, dass die Bürger zwingend auf den Rat der Experten angewiesen sind, da sonst "eine Ausweitung des Vermögens zur Entscheidungsfindung auf eine wachsende Zahl derjenigen stattfinden würde, die dafür nicht kompetent sind" (Bobbio, zitiert in Zolo, 1992: 103, Übersetzung des Verfassers).

Die sogenannten realistischen Theoretiker der Demokratie stellen also die

7) Der Begriff der pluralistischen Stagnation wurde vor allem in der angelsächsischen Literatur verwendet, so z.B. in Samuel H. Beers Abhandlung Britain Against Itself von 1982. Ähnliche Ideen erlebten aber unter dem Begriff des "Reformstaus" am Ende der ersten Regierung Schröder eine Renaissance. Zu diesem Zeitpunkt, d.h. zwischen dem Rücktritt von Oskar Lafontaine als Bundesfinanzminister im Jahr 1999 und dem Beginn der Hartz-Reformen nach Schröders knapper Wiederwahl im Jahr 2002 wurden sogenannte "Traditionalisten" in der SPD und den Gewerkschaften als Blockierer angegriffen und Schröder zu einer dezisionistischen Haltung aufgerufen. Schröder folgte diesen Forderungen mit seiner Regierungserklärung vom 14. März 2003, welche die Hartz-Reformen einleitete. Ein weiteres dezisionistisches Element seiner Politik waren die verschiedenen Expertenkommissionen, die Reformprogramme entwarfen, welche Schröder dann bei Gefallen ohne lange Debatten durch das Parlament schleusen konnte. 
Handlungsfähigkeit des Systems in den Mittelpunkt ihrer Betrachtung. Sie vertrauen darauf, dass demokratische Systeme durch "Polyarchien", d.h. miteinander konkurrierende Eliten in einem Gleichgewicht gehalten und permanent erneuert werden. Diese Eliten werden dabei "durch das Parteiensystem geschützt und legitimiert (...). Diese Gruppen, die in die vielfältigsten sozialen Sektoren eingebunden sind, wissen besser als jede "numerische Demokratie" die diffusen Anforderungen einer modernen Industriegesellschaft zu verstehen und auszudrücken, in der alle Primärinteressen und ein Großteil der legitimierten Interessen in der Lage sind, sich selbst zu organisieren" (Zolo, 1992: 110, Übersetzung des Verfassers).

Dieser Grundoptimismus der Demokratierealisten wird andererseits von den Theoretikern der demokratischen Partizipation in Frage gestellt. Sehen die Realisten das demokratische System hauptsächlich durch eine gegenseitige Blockade der Interessengruppen, also durch eine "Überfüllung" bedroht, beklagen die Unterstützer eines hohen Grades an demokratischer Partizipation stattdessen eine zunehmende Entleerung der Demokratie. Ausgangspunkt ihrer Kritik ist die Feststellung, dass Interessengruppen je nach Grad ihrer schon vorhandenen Machtressourcen innerhalb der demokratischen Institutionen systematisch unterschiedlich behandelt werden. Die mächtigsten wirtschaftlichen Interessen und die etablierten kulturell-sozialen Organisationen dominieren auch die politischen Parteien und genießen dabei staatliche Protektion. Der Wettbewerb zwischen den Gruppen wird durch bereits vorhandene Machtnetzwerke wie z.B. die staatlich und privat kontrollierten Medien, die staatlichen und halbstaatlichen Bürokratien, Privatfirmen, die Geheimdienste und andere nicht oder kaum sichtbare Kräfte dominiert. Obwohl die parlamentarische Demokratie oberflächlich betrachtet durch die Parteien kontrolliert wird, sind diese ihrerseits dem Einfluss anderer Machtstrukturen unterworfen, die, zumindest von den einfachen Parteimitgliedern, kaum beeinflusst werden können. Insgesamt könnte man daraus ableiten, dass die "Polyarchien" sich von der Gesellschaft mehr und mehr abschotten und damit gerade nicht für die Zirkulation und prinzipielle Offenheit des demokratischen Systems stehen können.

Die Anhänger der partizipatorischen Demokratie argumentieren also, dass das 
demokratische System von einer zunehmenden Zugangsschließung und einem gleichzeitigen Abbau der innerhalb des Systems überhaupt noch verhandelten Fragen gekennzeichnet ist: "Wenn demokratische Performanz gemessen wird an den Kriterien, (a) wie umfassend der Bereich der gesellschaftlichen Belange ist, die zur demokratischen Entscheidung anstehen, (b) wie effektiv die demokratische Selbstbestimmung der Bürger (Volkssouveränität) tatsächlich ist und (c) in welchem Maß das Prinzip der Gleichheit umgesetzt ist, dann ist die jüngste Entwicklung in den etablierten Demokratien eindeutig negativ" (Blühdorn, 2013: 91).

Abschließend kann festgehalten werden, dass die Frage einer möglichen Krise der Demokratie sehr stark von den Normen und Werten bestimmt wird, die in die Analyse einfließen. Für Vertreter eines minimalen und realistischen Standpunktes kann der generelle Rückgang der Wahlbeteiligung und der allgemeine Vertrauensverlust in die politische Exekutive in OECD-Ländern und in der Bundesrepublik Deutschland noch keine Krise des demokratischen Systems begründen (Pharr et al., 2001; Merkel, 2009). Andererseits erklären die Unterstützer partizipatorischer Demokratiemodelle, dass der Rückgang der Teilnahmewilligkeit der Bürger als Krise gewertet werden muss und dass ein Umschlagen in eine allgemeine Systemkrise zumindest nicht ausgeschlossen werden kann. Einer der dabei häufig diskutierten Faktoren ist die zunehmende Entkoppelung der Parteien von ihren historischen Milieus und der Rückgang der Parteimitgliedschaften, die im nächsten Abschnitt behandelt werden.

\section{Die Erosion der Volksparteien}

Seit der Vereinigung Deutschlands ist die Krise der Volksparteien ein Dauerthema, da die beiden traditionellen Volksparteien CDU/CSU und SPD seit 1990 kontinuierlich an Mitgliedern verloren. So büßte die CDU in diesem Zeitraum 39,7 Prozent ihrer Mitgliedschaft ein und hatte 2012 noch 313.222 Mitglieder, die CSU (die bayrische Schwesterpartei der CDU) verlor 20,5 Prozent und hatte 2012 noch 147.965 Mitglieder, die SPD verlor sogar 49,4 Prozent und hatte 2012 noch 466.365 Mitglieder 
(Niedermayer, 2013: 369). Ob diese Abwärtstrends mit dem Terminus "Krise" bezeichnet werden sollten, ist jedoch umstritten. So wird der Abstieg der Volksparteien z.B. auch als "Erosion" charakterisiert, da, anders als im Falle einer Krise, nicht mehr realistisch davon ausgegangen werden kann, dass die Rückgewinnung des früheren Mitgliederniveaus noch möglich wäre (Niedermayer, 2009: 112).

Andere Beobachter sehen den Mitgliederverlust der Volksparteien als eine Normalisierung bundesdeutscher Zustände mit vergleichbaren westeuropäischen Ländern wie z.B. den Niederlanden an, wo der langsame Abstieg der Christ- und Sozialdemokraten auf das Niveau "mittelgroßer Parteien" und die Entwicklung eines "nivellierten Vielparteiensystems" schon seit Anfang der siebziger Jahre durchlaufen wurde (Nijhuis, 2010). Der Abstieg der Volksparteien ähnelt im Übrigen auch dem Einfluss - und Mitgliederverlust anderer traditioneller Großorganisationen in Deutschland, z.B. den Kirchen, Arbeitgeberverbänden, Gewerkschaften und Sozialverbänden (Streeck, 2009: $46-55) .8)$

Um die gegenwärtige Situation der (ehemaligen) Volksparteien in Deutschland zu analysieren, muss darum zunächst das Konzept "Volkspartei" klarer gefasst werden. Hier gibt es jedoch in der Literatur sehr unterschiedliche Positionen. Einerseits wird argumentiert, dass die Existenz einer (relativen) Mehrheitspartei mit einem Stimmenanteil von mindestens 30 Prozent mit dem Konzept einer Volkspartei gleichgesetzt werden kann und dass darum die meisten westeuropäischen Länder mindestens eine, manche auch zwei solcher Parteien aufweisen (Smith, 1989: 158). Andererseits wird betont, dass das Konzept der Volkspartei in Deutschland umfassender gefasst werden sollte und als ein Sondertyp des Parteiensystems ("distinct entity") verstanden werden muss (Zolleis und Wertheimer, 2013: 98). Die drei besonders relevanten theoretischen Ansätze zur besseren Bestimmung der Rolle der Volksparteien

8) Keine Regel ohne Ausnahme: Der Allgemeine Deutsche Automobil-Club (ADAC) meldete 2013 einen neuen Mitgliederrekord. Dieser Zuwachs lässt sich vermutlich auf die Verdrängung anderer Automobilclubs vom Markt für Pannen- und Abschlepphilfen zurückführen. Allerdings funktioniert der politische Markt nach anderen Kriterien. 
sind dabei: 1. das Konzept der "Mehrheitspartei" (Smith, 1989), 2. die Idee der "Allerweltspartei" bzw. der "catch-all-party" (Kirchheimer, 1965, 1966) und 3. die Analyse der Rolle der Volks- als Mitgliederpartei (Wiesendahl, 2006).

Im Konzept der Mehrheitspartei ist bei der Bestimmung einer Volkspartei die wahlpolitische Verankerung der wichtigste Faktor. Aus dieser Sicht hat die CDU/ CSU ihren Status als Volkspartei bisher halten können, trotz allgemein sinkender Wahlbeteiligung, die auch die Union treffende Rückgänge in der Wahlzustimmung durch den hohen Prozentsatz an Wählerstimmen verdeckte. Die SPD ist so gesehen seit 2009 keine Volkspartei mehr und hat in mehreren Bundesländern (Bayern und in drei der fünf ostdeutschen Bundesländern) wegen ihrer geringen Stimmenanteile diesen Status auch in der Vergangenheit nie erreicht.

Abgesehen von der Wahlzustimmung würde dagegen der Rückgriff auf Kirchheimers Idee der Allerweltspartei erlauben, weiterhin beide Großparteien (Union und SPD) als zumindest potentielle Volksparteien zu bestimmen, da sie sich um die Zustimmung breiter Wählergruppen bemühen, dabei ihre Programmatik beständig an den gesellschaftlichen Wandel anpassen und ihre traditionelle Stammwählerschaft (konfessionell gebundene Wähler im Fall der CDU/CSU und gewerkschaftlich organisierte Arbeiter im Fall der SPD) an innerparteilichem Einfluss eingebüßt hat. Union und SPD zeigen sich auch durch die Professionalisierung ihrer Führungsgruppen und deren tendenzielle Abkoppelung von der Massenmitgliedschaft charakterisiert, die für Kirchheimer als wichtiger Indikator einer Transformation des früheren Typs der ideologisch festgelegten Partei und seiner Auflösung in die Allerweltspartei gelten. Allerdings ergeben sich aus Smiths und Kirchheimers Thesen auch Probleme aufgrund der mangelnden Trennschärfe ihrer Konzepte zwischen "Volksparteien" und "mittelgroßen Parteien." Es ist z.B. denkbar, dass eine Partei wie die SPD zwar die Wahlzustimmung als Volkspartei eingebüßt hat, jedoch den programmatischen Anspruch einer Volkspartei weiterhin aufrechterhalten möchte.

Die dritte theoretische Perspektive, die auf der Rolle der Volks- als Mitgliederpartei aufbaut, kann diese mangelnde Trennschärfe ebenfalls nicht auflösen. Obwohl es zutrifft, 
dass die deutsche Konzeption der Volkspartei die breite Mitgliedergewinnung in allen Volkschichten beinhaltetet - d.h. im Fall der CDU/CSU die Überwindung der alten Honoratiorenpartei und des "Kanzlerwahlvereins" der Adenauer-Periode und im Fall der SPD die Öffnung für Mitglieder aus den neuen Mittelschichten und religiös (insbesondere protestantisch) geprägte Milieus - so kann dieses Kriterium heute nicht mehr als Trennlinie zwischen Volks - und mittelgroßen Parteien dienen. Einerseits ergibt sich dies aus dem allgemeinen Mitgliederverlust der Parteien, der durch gesellschaftliche Individualisierung, den Wertewandel und die Abschwächung der Bindungskraft der den Parteien traditionell nahestehenden sozialen Milieus bedingt ist (Jun, 2011: 206-209; Linden und Thaa, 2011: 16). Noch wichtiger als der numerische Mitgliederverlust ist aber die soziale Schließung von Union und SPD gegenüber den benachteiligten Gruppen in der Gesellschaft. Deshalb überzeugt die Aussage, dass CDU/CSU und SPD eine hohe Zahl unterschiedlicher sozialer Schichten miteinander verbinden und neu entstehende Gruppen integrieren würden, immer weniger (Zolleis und Wertheimer, 2013: 101). Zwar versuchen Union und SPD eine programmatische Modernisierung und die Öffnung zu neuen Wählergruppen, jedoch werden solche Manöver durch Verluste bei den noch vorhandenen Stammwählern und Kernmilieus ausgeglichen (Walter et al., 2011: 163, 179, 218-219).

In diesem Zusammenhang muss auch betont werden, dass sich die noch vorhandene Mitgliedschaft von Union und SPD - im Gegensatz zum programmatischen Anspruch der Volksparteien - aus Repräsentanten einer privilegierten gesellschaftlichen Elite zusammensetzt. So besteht in der akademischen Literatur ein Konsens darüber, dass sowohl Union als auch SPD in erster Linie Mitglieder aus den höheren Einkommens-, Berufs- und Bildungsschichten rekrutieren (Niedermayer, 2009: 118, 122-123). Obwohl zwischen der Union und der SPD weiterhin bedeutende Unterschiede in der Sozialstruktur bestehen und die Parteimitgliedschaft von Selbstständigen in der Union einer Quelle zufolge bis zu viermal höher und die Zahl der Arbeiter weniger als halb so hoch ist wie im Fall der SPD, sind in beiden Parteien die Angehörigen der gehobenen Schichten in der Mehrheit. Die Mitgliedschaft der SPD scheint dabei sogar einen leicht 
höheren Anteil an Beamten und Angestellten aufzuweisen als die Union (Wiesendahl, 2006: 88-90).9) Es kann davon ausgegangen werden, dass diese soziale Exklusivität sich im Lauf der Zeit durch die Alterung der noch zu Hochzeiten der Volksparteien gewonnenen Mitglieder weiter verstärkt.

Die Konsequenz des politisch-organisatorischen Strukturwandels innerhalb von Union und SPD ist, dass sie sich, mehr oder weniger schnell, von Volksparteien in Parteien eines neuen Typs transformieren. Die Analyse dieses Prozesses verlangt, den dabei auftretenden Wandel in den Beziehungen zwischen Parteieliten, Mitgliedern und Wählern zu erklären. Hier fällt zunächst auf, dass dem neuen Parteityp im Vergleich zur traditionellen Volkspartei weniger Funktionen zuerkannt werden. Ideologische Fragen und Programmatik, die Artikulation und Aggregation von gesellschaftlichen Interessen sowie Sozialisierungsfunktionen der Parteien für ihre Mitglieder treten in den Hintergrund, während die direkte Kommunikation von Parteieliten mit den Wählern in professionellen und finanzintensiven Wahlkampagnen in den Vordergrund tritt. Der neue Parteityp wird also zunächst negativ als Ergebnis des Mitgliederund Einflussverlusts der Volksparteien gefasst. Was genau den neuen Parteityp ausmacht - und wie viel aus dem Erbe der Volksparteien in neuer Gestalt erhalten bleibt - ist jedoch weiterhin umstritten. So wurden z.B. Begriffe wie "professionelle Wahlpartei" (electoral-professional party) (Panebianco, 1988) oder "moderne Kaderpartei" vorgeschlagen (Koole, 1994)10), um die mitgliederärmeren und

9) Allerdings wird eine Analyse der sozialen Zusammensetzung der Parteimitgliedschaft dadurch erschwert, dass diese Daten meist nur einmal beim Eintritt in eine Partei erhoben werden, d.h. vorhandene Daten haben über längere Zeiträume nur geringere Aussagekraft (Niedermayer, 2009: 116-117).

10) Koole (1994) sieht eine Reihe von Kernelementen der "neuen Kaderpartei" (die Nähe zur Terminologie der leninistischen Kaderpartei ist dabei nicht beabsichtigt). Diese sind 1. die professionelle Führung, 2. die Dominanz der Parlamentsfraktion bzw. der Regierungsmitglieder (im Fall einer Regierungsbeteiligung), 3. die niedrigere Parteimitgliedschaft im Vergleich zur Periode der Volkspartei, 4. das Fortbestehen regionaler Parteiorganisationen, die allerdings nur selten (z.B. in Vorbereitung von Wahlen) aktiviert und sonst weitgehend durch informelle Parteinetzwerke ersetzt sind 
weitgehend professionalisierten Parteien zu beschreiben, die an die Stelle der Volksparteien treten oder getreten sind.

Darüber hinaus ist die tendenzielle Selbstermächtigung der Parteiführer und der in Parlamentsfraktionen tätigen Berufspolitiker auf Kosten der Parteibasis auch als "Kartellpartei" bezeichnet worden (Katz und Mair, 1995), weil solche Parteieliten für ihre materielle Existenz kaum noch auf Mitgliedsbeiträge angewiesen sind. An deren Stelle tritt die staatliche Parteienfinanzierung, d.h. die etablierten Parteien bilden in diesem Sinn ein Allparteienkartell, um die Finanzquellen und die Regeln des politischen Wettbewerbs zur gegenseitigen Befriedigung zu kontrollieren. Allerdings folgen diese Analysen im Wesentlichen der früheren Diagnose von Kirchheimer und setzten dessen Beschreibung des Niedergangs der Programm- und Massenpartei und ihrer Auflösung in eine Wahlstimmenmaximisierungspartei fort. Wenn es aber tatsächlich zutreffen würde, dass die Mitgliedschaft fast keinen Einfluss auf eine tendenziell geschlossene Parteiführung und die durch sie vertretene Politik mehr hat, stellt sich die Frage, welche Gründe es dann überhaupt noch für eine Parteimitgliedschaft geben sollte.

Eine Gegenströmung in der Literatur argumentiert deshalb auch, dass die Parteimitglieder selbst durch die zunehmende Professionalisierung transformiert werden, d.h. die Mitglieder reagieren auf die Umwandlung der Volkspartei in professionalisierte, wahlorientierte und mitgliederärmere Parteien durch verändertes Verhalten. Einerseits werden die traditionellen Regional - und Basisorganisationen der Parteien durch themenspezifische Netzwerke ergänzt, die es Parteimitgliedern ermöglichen, professionelle, themen - und gruppenspezifische Interessen innerhalb gesonderter Parteistrukturen zu verfolgen. Diese Entwicklung wird häufig mit dem Begriff der "Netzwerkpartei" charakterisiert (Heidar und Saglie, 2003). Allerdings sind solche Netzwerke nicht neu, d.h. sowohl Union als auch SPD entwickelten solche Strukturen schon zu Hochzeiten der Volkspartei der späten sechziger und frühen siebziger Jahre.

Zusätzlich versuchen die Parteiführungen neue Angebote zur direkten Einflussnahme der Parteibasis zu entwickeln, um dem Eindruck, dass die Parteien autoritär gelenkt 
würden, entgegen $\mathrm{zu}$ wirken. Jedoch haben weder Themennetzwerke noch die verschiedenen (und häufig eher symbolischen) Versuche einer die Parteibasis stärkenden direkten Einflussnahme auf die Parteilinie, wie z.B. ein "Mitgliederentscheid" der SPD über die Kanzlerkandidatur im Jahr 1993, den Mitgliederrückgang der Volksparteien stoppen können. Auch die direkte Einbeziehung der Mitglieder in neue Formen innerparteilicher Demokratie kann keine Antworten auf die Erosion der Volkspartei anbieten, wenn solche nach innen gerichteten Parteiaktivitäten unter der Teilnahmslosigkeit der Parteibasis leiden.11)

Die in Deutschland am stärksten vom Niedergang als Volkspartei betroffene SPD bemüht sich daher auch um eine Neubegründung ihrer Rolle. Allerdings findet sie in einem Grundsatzpapier ebenfalls keine überzeugenden Antworten. Zunächst wird an Selbstkritik nicht gespart. So heißt es mit klarem Bezug auf die Schröder-SPD der Jahre bis 2005, dass "reine Gefolgschaftsparteien, die sich um einen autoritären Führer gruppieren, der von kleinen Einflussgruppen gestützt und abgesichert wird und nur eine Willensbildung von oben nach unten zulassen, (...) kaum vielfältige und tiefe Wurzeln im gesellschaftlichen Leben schlagen. Sie können in diesem Sinne auch nicht Volkspartei sein" (Kommission Grundwerte, 2010: 4). Jedoch ist nicht klar, wie die SPD den Begriff der Volkspartei für sich definiert und wie weit sie sich noch als eine solche Partei versteht. Zwar wird festgehalten, dass die SPD den Anspruch beibehält, "führende Regierungspartei in einer Zweierkoalition werden zu können" (ibid.: 5). Wie dieser Anspruch eingelöst werden könnte, wird aber nicht erklärt. Scheinbar soll die SPD vor allem deshalb weiter Volkspartei sein, damit ihre Wahlkämpfe mit Aussicht auf Erfolg geführt werden können. So wird mit Blick auf die Frage des Verhältnisses zwischen Professionalisierung der Führung und Aktivitäten der Parteibasis ausgeführt, dass Volksparteien "lose verkoppelte" Organisationsstrukturen aufweisen. Diese erlauben

11) Die Probe aufs Exempel war in diesem Zusammenhang der Mitgliederentscheid innerhalb der SPD über die von SPD-Kanzler Schröder fast ohne Parteikonsultation durchgesetzte "Agenda 2010", der im Jahr 2003 das Quorum von 67.000 Mitgliederstimmen verpasste und darum von der Parteiführung nicht zur Abstimmung gestellt werden musste. Ein solcher SPD-Mitgliederentscheid hätte die Politik von Schröder theoretisch stoppen können. 
die "Gleichzeitigkeit verschiedener Organisationsrationalitäten (...). Eine Vereinigung der auf Mitglieder orientierten Massenintegrationspartei mit den Eigenschaften einer durch Eliten gesteuerten Wählerpartei wäre unter Umständen möglich. Sie ist auch notwendig (...). Analysen (...) zeigten, dass die professionalisierte, zentralisierte und über Medien vermittelte Wahlkampagnen [sic] zwar den Zweck der Überredung heterogener Wählerschichten erfüllten. Jedoch erwiesen sich klassische Mobilisierungskampagnen durch die lokalen Parteimitglieder als effektiver beim Stimmengewinn. Die notwendige Professionalisierung der Partei in einer Medien-Gesellschaft muss mit einer bedeutungsvollen Integration und Aktivierung der Mitglieder in Einklang gebracht werden" (ibid.: 9).

Allerdings scheinen die Autoren des Strategiepapiers selbst Zweifel an der Kombination aus Volks - und professioneller Wahlpartei zu haben. So räumen sie ein, dass die gesellschaftliche Fragmentierung zunimmt und erklären, dass "Bürgerinteressen (...) sich künftig immer stärker jenseits der Parteienlandschaft artikulieren. Das mag man bedauern, aufhalten werden wir diese Entwicklung in der Tendenz nicht" (ibid.: 11). Als Ergebnis dieser widersprüchlichen Überlegungen ergibt sich schließlich die Forderung, dass die SPD nur als Anführer eines Parteienlagers, das auch Grüne und Linke umfassen soll, wieder Volkspartei werden kann. In diesem Sinn sei der Begriff Volkspartei "als Charakterisierung derjenigen Parteien zu benutzen, die in der Lage sind, eines der beiden großen politischen Spektren, Mitte-Rechts oder Mitte-Links koalitionspolitisch zu organisieren und programmatisch zu prägen" (ibid.: 20). Diese mögliche Zukunft der SPD wird dann zum Abschluss auch noch als "die einzig realistische" eingeschätzt (ibid.). Wenn dem so ist, wird die SPD aber auch in Zukunft nur eine mittelgroße und weitgehend professionalisierte Partei sein können, denn die Anführerrolle eines Mehrparteienlagers bedeutet tendenziell die Aufgabe des Projekts einer breit aufgestellten Sozialdemokratie als Volkspartei. Dieses Problem der SPD wird im Schlussteil noch einmal aufgegriffen. Zunächst wird nun das Problem der sinkenden Wahlbeteiligung näher untersucht. 


\section{Die demokratische Beteiligungslücke}

Die Beteiligung an politischen Aktivitäten folgt im Allgemeinen einer Logik der Ressourcenausstattung, d.h. Bürger mit höherem Einkommen, höherer Bildung und fester Bindung an eine Region oder Stadt (z.B. durch den Besitz von Wohneigentum) sind im Allgemeinen politisch aktiver als Bürger, die in dieser Hinsicht geringere Ressourcen besitzen. Je höher der zeitliche Aufwand für politische Aktivitäten ist, umso ungleicher ist die Bereitschaft zur Teilnahme. Umgekehrt gilt auch, dass eine allgemein geringe Bereitschaft zu politischer Aktivität die Gruppe derjenigen, die trotzdem dazu bereit sind, weniger repräsentativ werden lässt (Lijphart 1997: 1-2). Politische Aktivitäten folgen also generell einer Logik, die Ungleichheit verstärken oder abschwächen kann: Je mehr Anforderungen an die Aktiven gestellt werden, umso mehr tendiert das politische System zur Exklusivität. Umgekehrt gilt, dass geringere Anforderungen die Bereitschaft zur Teilnahme erhöhen können. Da Wahlen weniger anspruchsvoll und zeitaufwendig sind als z.B. die Mitarbeit in Bürgerinitiativen, kommen sie dem demokratischen Ideal gleicher Teilhabe der Bürger am politischen System entgegen. Trotzdem nimmt auch die Bereitschaft zur Wahlteilnahme in fast allen OECDLändern seit mehreren Jahrzehnten tendenziell ab; so auch in der Bundesrepublik, wo die Wahlbeteiligung bei Bundes-, Landtags-und Kommunalwahlen seit den neunziger Jahren besonders stark gefallen ist (Schäfer und Streeck, 2013: 13). Die politisch relevante Frage ist, ob diese sinkende Wahlbeteiligung gleichmäßig in allen gesellschaftlichen Milieus und sozialen Schichten auftritt oder ob sich die Wahlenthaltung systematisch auf bestimmte Gruppen konzentriert, d.h. das Wahlergebnis systematisch verzerrt wird.

Zunächst muss betont werden, dass diese Frage ein in der Politikwissenschaft umstrittenes Thema betrifft. Es galt lange Zeit als erwiesen, dass sich Nichtwähler in ihren politischen Haltungen nicht systematisch von der Gruppe der Wähler unterscheiden, d.h. ihre politischen Präferenzen nicht oder nur gering von denen der Wähler abweichen (Verba et al., 2004: 648-649, 652). Ein Grund für die besondere 
Schwierigkeit der Bestimmung politischer Auswirkungen der Wahlenthaltung liegt infolgedessen zunächst in der hohen Zahl an möglichen Variablen begründet, welche die Wahrscheinlichkeit der Wahlbeteiligung beeinflussen können. So sind Faktoren wie die politische Kultur eines Landes, die Art des Wahlsystems (insbesondere die Auswirkung des Mehrheits- oder proportionalen Wahlrechts auf die Wahlbeteiligung), der Grad an sozialer Ungleichheit und individuelle Faktoren wie Alter, Erziehung, sozialer Status und Einkommen als relevante Variablen bei der Analyse der Teilnahmewahrscheinlichkeit an Wahlen (und anderen Formen der politischen Beteiligung) allgemein anerkannt (Schäfer, 2013). Was jedoch immer noch bezweifelt wird, sind systematische Auswirkungen einer sinkenden Wahlbeteiligung auf das Wahlergebnis. ${ }^{12)}$

In der relevanten Literatur finden sich darum auch vier widersprüchliche Hypothesen zur politischen Relevanz des Anstiegs der Nichtwähler, die man wie folgend zusammenfassen kann: 1. Die "Kein Grund zur Beunruhigung-These" geht davon aus, dass eine sinkende Wahlbeteiligung möglicherweise die Qualität der politischen Willensbildung erhöht, weil nur noch Besser-Informierte an Wahlen teilnehmen; 2. die "Neue Formen der politischen Beteiligung-These" erklärt, dass die Nichtteilnahme an Wahlen durch alternative Formen politischer Aktivität substituiert wird; 3. die "Strategische Neuausrichtung-These" erwartet, dass Parteien auf die sinkende Wahlbeteiligung durch eine Neuausrichtung ihrer Programmatik reagieren und sich dabei auf die sozialen Gruppen konzentrieren, die mit großer Wahrscheinlichkeit weiter zur Wahl gehen und 4. die "Soziale Selektion der Nichtwähler-These" geht davon aus, dass die benachteiligten Schichten sich zunehmend von der Teilnahme an Wahlen zurückziehen und das Wahlergebnis dadurch systematisch verzerrt wird (siehe Schäfer, 2011: 135-137 für weitere Literaturhinweise).

12) Empirische Forschungen leiden außerdem unter besonderen Schwierigkeiten bei der Gewinnung von Nichtwählern zur Teilnahme an Meinungsumfragen und dem Problem, dass Umfragen häufig in sozial erwünschter Form beantwortet werden, was die Ergebnisse möglicherweise verzerrt (Schäfer 2012: 243). 
Die erste These ist in ihrer Tendenz elitär und demokratiefeindlich, da sie bei steigender Nichtbeteiligung am politischen System und an Wahlen eine höhere Qualität der Willensbildung zu erkennen meint. Die zweite These einer Substitution der Wahlteilnahme durch neue Formen der politischen Beteiligung ist demokratiefreundlicher, findet aber in der empirischen Forschung keine Bestätigung. Tatsächlich sind Wähler eher bereit, auch an anderen politischer Aktivitäten teilzunehmen, während Nichtwähler politische Aktivitäten häufiger generell meiden, d.h. es entsteht eine Kluft zwischen den besonders Aktiven und den besonders Inaktiven. Die dritte These einer strategischen Neuausrichtung der Parteien auf die sozialen Milieus, die mit hoher Wahrscheinlichkeit an Wahlen teilnehmen, und die vierte These einer zunehmenden Segregation der politischen Beteiligung zwischen bessergestellten und benachteiligten Wählern treffen dagegen für die Bundesrepublik tendenziell zu, wie nun gezeigt werden wird.

Zunächst muss daran erinnert werden, dass beide traditionellen Volksparteien, die Union und die SPD, über längere Zeiträume an Wahlzustimmung eingebüßt haben. Die SPD hat jedoch deutlich höhere Verluste erlitten als die CDU/CSU. Ein Hauptgrund dafür besteht in der räumlichen Segregation zwischen bessergestellten und benachteiligten Wählern, die über längere Zeiträume (seit den siebziger Jahren) mit einer steigenden Streuung der Wahlbeteiligung einhergeht. In einfachen Wohnlagen, in denen die SPD historisch vermehrt gewählt wurde, ist die Wahlbeteiligung bereits in den siebziger Jahren schon etwas geringer als in besseren Wohnlagen, in denen die Union ihre Hochburgen hat. Jedoch hat sich diese ursprünglich noch recht begrenzte Streuung bis heute stetig verstärkt und kann nun treffend als demokratische Beteiligungslücke bezeichnet werden. In einfachen Wohnlagen gehen sehr viel weniger Menschen zur Wahl als in den mittleren und gehobenen: "Die Kenntnis des sozialen Profils von Stadtteilen ermöglicht relativ zuverlässige Aussagen sowohl über die Wahlbeteiligung als auch über das Abschneiden der Parteien" (Schäfer, 2012: 245). Um diese Trends abzubilden, müssen jedoch kleinere räumliche Einheiten, d.h. Stadtteile oder (falls datenmäßig vorhanden) noch kleinere Einheiten untersucht werden, da größere Einheiten die Effekte einer sozial segregierten Wahlbeteiligung 
tendenziell verdecken.

Aus einer Untersuchung kleiner räumlicher Einheiten, die eine höhere Homogenität der Sozialmilieus und Siedlungsformen aufweisen, lassen sich kausale Verbindungen zwischen sozialökonomischen Strukturdaten wie z.B. der Höhe der Arbeitslosigkeit und der Zahl der Empfänger von Transferleistungen einerseits und der Wahlbeteiligung andererseits aufzeigen. Ein sozial segregierter Rückgang der Wahlbeteiligung ist dabei politisch nicht neutral, sondern schadet besonders den Mitte-Links-Parteien: "SPD und Die Linke erzielen ihre besten Ergebnisse dort, wo die Wahlbeteiligung niedrig ausfällt, während die CDU und insbesondere die FDP in Vierteln mit hoher Beteiligung am besten abschneiden. Gerade dieses Muster erklärt, weshalb der Rückgang der Wahlbeteiligung linken Parteien schadet: In SPD-Hochburgen, so zeigt der Längsschnitt, ist die Wahlbeteiligung am stärksten zurückgegangen" (Schäfer, 2012: 241, Hervorhebung im Original; siehe auch Schäfer, 2011: 141-144). Andererseits trifft auch zu, dass eine insgesamt hohe Wahlbeteiligung den Mitte-Links-Parteien und insbesondere der SPD nützt, weil dann die Mobilisierungslücke zu den bürgerlichen Parteien geschlossen wird. Dies kann mit kleinräumigen Daten für Bremen und Köln, wo diese Daten über längere Zeiträume (seit 1972 und seit 1987) erhoben wurden, nachgewiesen werden.13) Es zeigt sich, dass die Wahlbeteiligung innerhalb dieser Städte in den kleinräumigen SPD-Hochburgen über längere Zeiträume systematisch stärker zurückging als in den kleinräumigen CDU-Hochburgen (d.h. asymmetrisch gefallen ist): je geringer die Wahlbeteiligung, desto schlechter das stadtweite SPD-Ergebnis und desto besser das stadtweite CDU-Ergebnis (Schäfer, 2012: 257).

13) Schäfer untersucht 34 deutsche Großstädte auf der Grundlage vorhandener Daten, woraus sich ein klares Bild ergibt: Die SPD ist die Verliererin der zunehmenden Wahlenthaltung in den einfachen innerstädtischen Wohnvierteln, während die Wahlbeteiligung in Vierteln mit höherer Wohnqualität weiterhin - und mit wachsender demokratischer Beteiligungslücke gegenüber benachteiligten Vierteln - stabil ist und Union, Grüne und FDP hier ihre besten Ergebnisse erzielen. Eine allgemein steigende Wahlbeteiligung verbessert dagegen das SPD-Ergebnis und im Fall der Stadt Bremen auch das Ergebnis der Linken (Schäfer, 2012: 257-260). Allerdings sind die Datenquellen unvollständig und betreffen überwiegend traditionelle SPD-Hochburgen (ibid.: 250). 
Zusammenfassend zeigt die Verwendung kleinräumiger Daten Anhaltspunkte dafür, dass die sinkende Wahlbeteiligung aufgrund der ungleichen räumlichen Verteilung der Nichtwähler und ihrer Konzentration in sogenannten städtischen Problemvierteln häufig früheren Arbeitervierteln und SPD-Hochburgen - eine direkte Auswirkung auf das Wahlergebnis haben kann. Diese Aussage muss selbstverständlich durch weitere Studien untermauert werden. Allerdings soll hier keine Bilanz eines innovativen Forschungsfeldes zur Erklärung der Dauerkrise der SPD gezogen werden, sondern abschließend die Idee der demokratischen Beteiligungslücke in Bezug auf zwei Einwände verteidigt werden.

Der erste Einwand betrifft die Frage, ob die Krise der SPD konjunkturell oder strukturell bedingt ist. Einige Autoren bemühen sich z.B. weiterhin um konjunkturelle Erklärungen und verweisen auf Faktoren wie die fehlende Attraktivität der letzten beiden SPD-Kanzlerkandidaten, welche die Wähler abgeschreckt habe. So wird argumentiert, dass "weniger die soziodemographischen Faktoren eine Rolle spielen, als vielmehr die Einstellungen zu den Parteien bzw. deren Repräsentanten" (Kulick, 2011: 213). Jedoch müssen solche konjunkturellen Faktoren - trotz unbestritten hohem Einfluss auf einzelne Wahlen - letztlich doch im Rahmen der besonderen strukturellen Nachteile der SPD interpretiert werden. Die Stärke der Untersuchung der Wirkung struktureller Faktoren und einer Datenauswertung über längere Zeiträume besteht gerade darin, dass sie der demokratischen Beteiligungslücke und der Krise der SPD ein Gesicht gibt. Das SPD-Milieu ist tendenziell weniger stabil und weniger geschlossen als das Milieu der Union und leidet besonders stark unter soziokulturellem Veränderungsdruck. ${ }^{14)}$ Um mit der Union auf Augenhöhe zu konkurrieren, muss die

14) Die Union erfreut sich traditionell der Unterstützung des katholischen Milieus, insbesondere regelmäßiger Kirchgänger, unabhängig davon, ob es sich um Arbeiter, Angestellte, Beamte oder Selbstständige handelt. Außerdem erhält sie hohe Zustimmung im Mittelstand, bei Selbstständigen und in der Landwirtschaft. Die SPD wird dagegen historisch von Arbeitern und gewerkschaftlich organisierten Arbeitnehmern unterstützt, deren Zahl tendenziell sinkt. Obwohl die Wählerbindung an die Parteien generell abnimmt, ist das Unionsmilieu weiterhin größer und auch stabiler als das der SPD. 
SPD "wesentlich mehr Integrations - und Kommunikationsleistungen erbringen", und dabei ihre Stammwähler halten und Wechselwähler von anderen Parteien - wegen der Größenverhältnisse primär von der Union - sowie ehemalige Nichtwählern oder Jungwähler anziehen (Stöss und Neugebauer, 1998: 7, 9). Eine erfolgreiche Wahlmobilisierung der SPD verlangt darum eine Allianz - oder auch einen Spagat zwischen verschiedenen Milieus; insbesondere muss in der Gruppe der Arbeitnehmer mit der Union und der Linken und in der Gruppe der postmateriellen Mittelschichten mit den Grünen konkurriert werden. Diese besonderen Strukturprobleme der SPD bleiben jedoch bei einer Analyse, die vor allem auf Kandidaten- und Themenkonkurrenz aufbaut, unberücksichtigt.

Der zweite Einwand betrifft die Frage, ob die SPD nicht Teile ihres alten Stammwählerpotentials in den sozial benachteiligten Schichten aufgeben muss, um in Konkurrenz zur Union die Wechselwähler der Mitte für sich zu gewinnen. Eine solche Orientierung der SPD auf das politische Zentrum ist implizit und explizit der Kern der "neuen" Sozialdemokratie, wie sie in Deutschland zunächst durch Schröder und den sogenannten Agenda-Flügel der SPD zwischen 1999 und 2005 und seit dieser Zeit in abgeschwächter Form durch das SPD-Nachfolgequartett Gabriel-Nahles-Steinbrück -Steinmeier verfolgt wurde. Eine Distanzierung der SPD von den absteigenden oder bereits abgestiegenen sozialen Gruppen in der Bundesrepublik folgt der Logik des politischen Wettbewerbs, die auf die abnehmende Wahlbeteiligung in den Problemvierteln durch strategische Neuausrichtung auf die aufsteigenden Milieus reagieren möchte (wie in der dritten und vierten These weiter oben bereits ausgeführt). Um dies ohne Selbstzweifel tun zu können, wird von SPD-nahen Sozialforschern deshalb immer wieder die relative Ferne der benachteiligten Schichten vom SPD-Milieu behauptet. Allerdings stellen diese Analytiker durch eine soziologische Feineinteilung des materiell unterprivilegierten sozialen Drittels der deutschen Gesellschaft in drei voneinander kulturell und politisch getrennte Sub-Milieus, nämlich die "Selbstgenügsamen Traditionalisten", die "Autoritätsorientierten Geringqualifizierten" und das "Abgehängte Prekariat", diese Distanz - zumindest teilweise - erst her und überbetonen sie somit 
stark (Neugebauer, 2008).15)

Was hier jedoch fehlt, ist ein Bewusstsein für die Mobilisierungsnotwendigkeiten der SPD bei den benachteiligten Gruppen. So wird der besondere Mobilisierungsrückstand der SPD bei den vom sozialen Abstieg bedrohten Arbeitnehmern und den "Abgehängten Prekären" in SPD-Studien zwar festgestellt, die Frage einer Rückgewinnung dieser Gruppen aber überhaupt nicht mehr aufgeworfen (Kommission Grundwerte, 2010: 13).16) Tatsächlich ist die SPD aber - selbst aus rein wahlpolitischen Motiven - mit dieser freiwilligen Überantwortung der sozial benachteiligten Schichten an andere Parteien nicht gut beraten, denn die strukturellen Mobilisierungsvorteile der Union in den sozial besser gestellten Milieus sind weiterhin vorhanden (Dostal, 2012: 298-299). So scheint es daher, als habe die SPD einige ihrer traditionellen Wählergruppen freiwillig aufgegeben und sich damit dauerhaft mit dem Status einer nur noch mittelgrossen Partei arrangiert. Im nächsten Abschnitt wird die theoretische Debatte mit Bezug auf die SPD zusammengefasst.

\section{Die Dauerkrise der SPD}

Dieser Abschnitt skizziert zunächst die von der SPD in den beiden rot-grünen Regierungen (1998-2002 und 2002-2005) und dann in der Rolle des Juniorpartners

15) Diese Terminologie folgt weitgehend den Marktforschern des Sinus-Instituts (2011: 14), die die Sozialmilieuanalyse in der deutschen Soziologie popularisierten. Neugebauer ordnet die Gruppe der Traditionalisten tendenziell der Union zu, verortet die Prekären hauptsächlich bei den Linken und den rechtspopulistischen Parteien und stellt einen annähernden Gleichstand von Union und SPD in der Zustimmung bei Geringqualifizierten fest (2008: 34-35).

16) In der Studie der Kommission Grundwerte (2010) werden z.B. Daten des SPD-Parteivorstands aus dem Jahr 2007 zitiert, die eine Mobilisierungslücke (die in Meinungsumfragen ausgewiesene Lücke zwischen tatsächlichen und potentiellen SPD-Wählern) in der "bedrohten Arbeitnehmermitte"von 17 Prozent und in der Gruppe des "Abgehängten Prekariats" von 21 Prozent ausweisen. In keinem der anderen acht untersuchten Sozialmilieus ist der Mobilisierungsrückstand der SPD größer. 
in der Großen Koalition mit der Union (2005-2009) verfolgte Politik. Dann wird der sich daraus ergebende Niedergang bei Wahlen noch einmal analysiert und abschließend festgestellt, dass die SPD ihre Dauerkrise nicht überwunden hat.

Zunächst muss daran erinnert werden, dass die SPD 1998 mit dem Wahlslogan "Innovation und Gerechtigkeit" ihren größten Wahlerfolg erzielen konnte. Die darauf folgende Ablösung der Kohl-Regierung durch die rot-grüne Koalition überdeckte aber die Tatsache, dass es innerhalb der SPD keine Übereinstimmung hinsichtlich der Regierungsstrategie gab. So war die Ambiguität des Verhältnisses zwischen dem stramm markt - und unternehmensfreundlich auftretenden Schröder ("Innovation") und dem eher als keynesianisch und solidaristisch-umverteilend ausgewiesenen Oskar Lafontaine ("Gerechtigkeit") im Wahlkampf eine Stärke, weil auf diese Weise scheinbar beide Parteiflügel zu ihrem Recht kommen konnten. Sobald die SPD aber die Regierung übernahm, zeigte sich der völlige Fehlschlag dieses Spagats: Lafontaine fiel als Finanzminister schon 1999 mit seiner Politik einer transnationalen Re-regulierung der internationalen Finanzmärkte und einem keynesianisch inspirierten Konjunkturprogramm durch und wurde nach einer gegen ihn forcierten Medienkampagne aus der Regierung gejagt. Für den Rest der Legislaturperiode hatte der Schröder-Flügel der SPD das Heft fest in der Hand, versuchte aber weiterhin eine Politik des Durchlavierens und der begrenzten wirtschaftlichen Reformschritte, die Schröder mit der Formel einer "Politik der ruhigen Hand" beschrieb. Jedoch kam es zwischen 1998 und dem Wahljahr 2002, in dem Rot-Grün nur mit Glück - einer erfolgreich gemeisterten Flutkatastrophe in Ostdeutschland, dem Heraushalten Deutschlands aus dem Irak-Konflikt und dem schwachen Unionskanzlerkandidaten - die Wahlen knapp für sich entscheiden konnte, zu einem stetigen Anwachsen einer neoliberal-marktorientierten politischen Hegemonie in Deutschland. Diese betraf beide Großparteien - SPD und Union - in hohem Maße. Sie wurde durch eine Kombination aus realen Problemen, d.h. einer stetig zunehmenden Massenarbeitslosigkeit und einer parallel dazu stattfindenden Elitenkoordination zur Durchsetzung eines neoliberalen Meinungsklimas (Alphajournalisten, Arbeitgeberlobby und eine neue neoliberal eingestellte Politikergeneration in allen Parteien mit Ausnahme 
der PDS) hergestellt.

Innerhalb der SPD fanden nun zwei grundlegende Veränderungen statt: Einerseits wurden Ideen einer keynesianischen Wirtschaftssteuerung als im Zeitalter der Globalisierung unwirksam verworfen, andererseits wurde eine Orientierung auf die Märkte (mit oder ohne Rückgriff auf den ursprünglich durch die CDU/CSU vertretenen Ordoliberalismus) zur neuen sozialdemokratischen Leitidee erklärt (Nachtwey, 2007). Diese neue Politik wurde nach der Wahl 2002 durch die Agenda 2010 und die Hartz-Reformen energisch durchgesetzt. Dabei war Schröders neue Linie klar angebotsorientiert und neoliberal inspiriert. Die Koordinierung der Regierungspolitik mit den Gewerkschaften wurde aufgegeben. Es folgte eine Politik des Sozialabbaus und der Deregulierung des Arbeitsmarktes. Diese betraf zeitliche Einschränkungen beim Arbeitslosengeld und verschärfte Bedürftigkeitsprüfungen bei Sozialleistungen an Langzeitarbeitslose. Zusätzlich wurden der Niedriglohnsektor und die zeitlich begrenzten Arbeitsverhältnisse massiv ausgeweitet. Die neue Strategie schien dem Motto "Augen zu und durch" zu folgen: Es wurde erwartet, dass die Gesellschaft die Folgen des Sozialabbaus bis zum nächsten Wahltag schon wieder vergessen haben würde.

Diese Hoffnung der SPD-Führung erwies sich aber als unzutreffend. Stattdessen entstand in der Zivilgesellschaft eine spontane Volks - und Massenbewegung gegen die Hartz-Reformen, die sich ohne institutionelle Anbindung oder Unterstützung der etablierten politischen Kräfte schnell zur größten außerparlamentarischen Mobilisierung in der Geschichte des vereinigten Deutschland entwickelte. Die Anti-Hartz-Bewegung (zentraler Slogan: "Weg mit Hartz 4, das Volk sind wir") richtete sich in erster Linie gegen Kürzungen von Sozialleistungen an Langzeitarbeitslose. Allerdings wurden durch die im Rahmen der Hartz-Reformen gleichzeitig stattfindenden Kürzungen bei den Kurzzeitarbeitslosen (Senkung der Bezugszeit des Arbeitslosengeldes) auch bei (noch) sozial integrierten Arbeitnehmern Ängste geweckt, sodass die Bewegung auch aus dieser Richtung steigende Unterstützung genoss. ${ }^{17)}$

17) Die Hauptauswirkung der Politik der angebotsorientierten Arbeitsmarktreformen war ein erhöhter 
Politisch war die SPD hauptverantwortlich für die Arbeitsmarktreformen, obwohl diese nur durch eine informelle große Koalition mit der Union, die in der für die Verabschiedung der meisten Gesetze notwendigen zweiten Parlamentskammer - dem Bundesrat - die Mehrheit kontrollierte, nach 2003 gemeinsam durchgesetzt werden konnten. Dabei ergab sich zwischen den zwei Parteien ein Wettlauf um die radikaleren Ansätze, da sich auch die Union auf ihrem Leipziger Parteitag 2003 stark neoliberal ausrichtete. Beide Parteien hofften, durch die gemeinsame Verantwortung für die Liberalisierungspolitik einer Abstrafung durch die Wähler zu entgehen. Jedoch schloss sich das Einfallstor für die neoliberalen Reformen nach 2003 aus drei Gründen langsam wieder. Erstens wurden die Politiker vom Ausmaß der Proteste und des Volkszorns gegen die Liberalisierungspolitik überrascht. Zweitens waren Meinungsumfragen zufolge die Sozialstaatskürzungen bei einer klaren Mehrheit der Deutschen - und insbesondere bei SPD-Anhängern - unpopulär. Drittens brachten die Ergebnisse der Bundestagswahl 2005 für beide große Parteien starke Wahlverluste. Da die Union sich in der Wahlkampagne 2005 für ein flat tax-Modell im Steuerrecht aussprach, sich also noch klarer neoliberal exponierte, verlor sie beinahe so viele Stimmen wie die SPD. Trotzdem wurde nach 2005 in der sich anschließenden Großen Koalition unter der Führung von CDU-Kanzlerin Merkel noch ein weiterer Einschnitt - die Anhebung des Renteneintrittsalters von 65 auf 67 Jahre - durchgesetzt. Dabei zeichnete der SPD-Vizekanzler Franz Müntefering, der Schröder nach dessen Wahlniederlage als SPD-Vorsitzender nachgefolgt war, als Arbeitsminister verantwortlich für das erhöhte Renteneintrittsalter, sodass die SPD einmal mehr die Hauptverantwortung für Kürzungen schulterte.

Rückblickend war mit der Wahl 2005 die neoliberale Welle in Deutschland erst

Druck auf die Beschäftigten, sich mit Lohnstagnation und einer Senkung von Sozialstandards abzufinden. Die Lohnstückkosten sind in Deutschland im EU-weiten Vergleich seit 2000 am wenigsten gestiegen und der deutsche Außenhandelsbilanzüberschuss nahm stark zu (Europäische Kommission, 2013). 
einmal gebrochen, denn die starken Verluste der beiden Großparteien erzwangen erste Korrekturen an den zuvor durchgeführten Reformen. Außerdem wechselten einige der führenden Vertreter des Liberalisierungskurses von der Politik in die Wirtschaft, was den Ton politischer Debatten sofort in Richtung einer moderateren Politik der Mitte zurückfallen ließ.

Aus dieser knappen Zusammenfassung der SPD-Politik seit 1998 ergibt sich die Folgerung, dass die Partei eine doppelte Strukturkrise durchmacht. Einerseits hatte sie - wie auch der Rest der europäischen Sozialdemokratie - keine eigenen Strategiemodelle zur Bewältigung der Globalisierung und des gesellschaftlichen Strukturwandels anzubieten und folgte deshalb Ideen, die aus einer markt- und neoliberalen Richtung kamen. Andererseits war eine solche Politik aber nur bei bestimmten Eliten Mainstream-Medien, Arbeitgebern und der FDP - wirklich mehrheitsfähig. In beiden Großparteien hingegen ergaben sich vor allem auf der Ebene der Massenmitgliedschaft und bei der Wählerschaft Vorbehalte gegenüber radikalen Einschnitten im Sozialstaat sowie hinsichtlich der Deregulierung des Arbeitsmarktes. Die Hoffnung der SPDParteiführung auf eine schnelle Überlagerung der sozialen Kosten der Reformpolitik durch andere politische Themen erwies sich daher als Illusion. Der Grund dafür war, dass traditionell der SPD nahestehende Wählergruppen die Hauptleidtragenden der Reformen waren. Ihre Erwartungen an den Sozialstaat wurden massiv zurückgeschraubt und der Druck auf die Arbeitsbedingungen der Stammbelegschaften in den Unternehmen wurde durch die Expansion der Zeitarbeit und anderer flexibilisierter Erwerbsformen stark erhöht. Dagegen waren die Gewinner der Reformen fast ausschließlich im Arbeitgeberlager zu finden, d.h. bei Gruppen, die der Union und nicht der SPD nahestehen.

Es stellt sich die Frage, warum die SPD-Führung, trotz der sich direkt an die AgendaPolitik anschließenden Serie dramatischer Wahlniederlagen, keine Kurskorrektur durchführte. Hier muss zunächst die Stärke der neoliberalen Hegemonie betont werden, die sich während der rot-grünen Regierungsjahre - auch bei den Grünen - herauszubilden begann. War die Kohl-Regierung noch der Tradition des Sozialstaats und der Interessenkoordinierung zwischen den Sozialpartnern verpflichtet, fand in den 
rot-grünen Jahren ein dramatischer Stimmungsumschwung statt. Die Globalisierung erschien in Deutschland als eine Bedrohung, der man nur durch Anpassung an den internationalen Mainstream und nicht durch eine Weiterentwicklung des deutschen Modells einer auf Koordinierung und Konsens beruhenden Marktwirtschaft beikommen konnte. Die engere SPD-Führung schien mehrheitlich die neoliberale Politik voll verinnerlicht $\mathrm{zu}$ haben und war davon überzeugt, dass die Partei für Modernisierungsleistungen zum Nutzen des Wirtschaftsstandorts Deutschland nach einer kurzen Durststrecke bei Wahlen wieder belohnt werden würde. In diesem Zusammenhang waren die Kontakte der SPD-Führung mit dem Arbeitgeberlager verbessert und die mit dem Gewerkschaftslager schon seit längerer Zeit zurückgefahren worden (Nawrat, 2012: 22, 88-103). Allerdings erwies sich dieser neue Realismus als politisch kostspielig, denn die SPD liquidierte Teile ihrer politischen Verankerung in den sozial benachteiligten Schichten, wurde dafür von den Wählern der Mitte jedoch nicht belohnt: Weder bei der Mobilisierung ihres Stammwählerpotentials noch bei der Gewinnung von Wechselwählern war der SPD Erfolg beschieden. Stattdessen resultierte ein "völliger Identitätsverlust: Wie ein auslaufendes Sieb verlor die SPD Wähler nach allen Seiten" (Niedermayer, 2010: 232).

Auch in der Opposition zwischen 2009 und 2013 gelang der SPD keine Erholung von den Folgen der Agenda- und Hartz-Politik. Alternative Ideen jenseits der marktorientierten Sachzwänge waren weiterhin nicht vorhanden, nur das alte Führungspersonal der Agenda-Jahre war entweder abgewählt worden (Schröder) oder schied aus Altersgründen aus der Politik aus (Müntefering). Die beiden anderen führenden Agenda-Repräsentanten wurden den Wählern nacheinander als SPD-Kanzlerkandidaten angeboten und erreichten sowohl 2009 (Franz-Walter Steinmeier) als auch 2013 (Steinbrück) die beiden schlechtesten Wahlresultate der SPD-Geschichte in der Bundesrepublik. Selbst unter neuer Führung wird die SPD darum wohl alten Wegen folgen. Mit dem Eintritt in eine weitere Große Koalition mit der Union nach der Bundestagswahl 2013 akzeptiert die Partei, dass sie in einer asymmetrischen Beziehung als Juniorpartner der Union gefangen ist. Alternativen sind zumindest kurzfristig keine 
vorhanden, denn weder "Rot-Grün" noch ein um die Linke erweitertes MitteLinks-Lager haben gegenwärtig eine gesellschaftliche Mehrheit. So wird es die SPD auch weiterhin schwer haben, auf politische Sachzwänge wie z.B. die Dauerkrise der Staatsfinanzen in Zeiten der Banken- und Eurorettungspolitik mit mehr als mechanischer Anpassung zu reagieren.

\section{Fazit}

Die Bundestagswahl 2013 ergab drei Hauptresultate, nämlich einen Wahlerfolg der CDU/CSU, eine sehr geringe Wahlbeteiligung und die Fortsetzung der Dauerkrise der SPD. Zwischen Union und SPD hat sich der Abstand bei der Wahlunterstützung weiter erhöht. Während die CDU/CSU ihre Rolle als breit aufgestellte Volkspartei - wenn auch in erster Linie auf Kosten des ehemaligen Koalitionspartners FDP und im Rahmen einer niedrigen Wahlbeteiligung - tendenziell beibehalten konnte, ist die SPD nur noch als mittelgroße Partei zu bezeichnen.

Diese Asymmetrie zwischen den beiden Hauptkräften des deutschen Parteiensystems erklärt sich aus dem wahlpolitischen Niedergang der SPD, der durch die Politik der neoliberal ausgerichteten rot-grünen Arbeitsmarktreformen seit 2003 ausgelöst wurde. Die primär durch die SPD zu verantwortenden Sozialkürzungen richteten sich gegen Teile ihrer Stammwählerschaft in den sozial benachteiligten Schichten. Diese Gruppen reagierten auf den Sozialabbau mit Wahlenthaltung oder der Wahl anderer Parteien und konnten durch die SPD auch in der Oppositionsrolle zwischen 2009 und 2013 nicht zurückgewonnen werden. Die Analyse verschiedener theoretischer Erklärungsmodelle (Krise der Demokratie, Erosion der Volksparteien und die wachsende demokratische Beteiligungslücke, d.h. die steigende Wahlenthaltung in ehemaligen innerstädtischen Hochburgen der SPD) ergibt, dass die Sozialdemokratie die ihr historisch nahestehenden sozialen Gruppen demobilisiert hat, ohne dafür bei 
den neuen Mittelschichten kompensatorische Wahlerfolge erzielen zu können. Die SPD ist darum keine Volkspartei mehr.

\section{Literaturverzeichnis}

Blätte, Andreas. "Reduzierter Parteienwettbewerb durch kalkulierte Demobilisierung. Bestimmungsgründe des Wahlkampfverhaltens im Bundestagswahlkampf 2009." Korte, Karl-Rudolf (Hrsg.). Die Bundestagswahl 2009. Analysen der Wahl-, Parteien-, Kommunikations - und Regierungsforschung. Wiesbaden: VS Verlag, 2010, S. 273-297.

Blühdorn, Ingolfur. Simulative Demokratie. Neue Politik nach der postdemokratischen Wende. Frankfurt/M.: Suhrkamp, 2013.

Bundeswahlleiter a. "Wahlkreise bei der Bundestagswahl 2013, in denen die Mehrheit der Erststimmen von 2009 zu 2013 auf eine andere Partei übergegangen ist." 4. Oktober 2013, http://www.bundeswahlleiter.de/de/bundestagswahlen/ BTW_BUND_13/veroeffentlichungen/ergebnisse/arbtab5.pdf (Stand: 23.12. 2013).

Bundeswahlleiter b. Wahl zum 19. Deutschen Bundestag am 23. September 2013. Ergebnisse früherer Bundestagswahlen. Berlin: Bundeswahlleiter, Juli 2013, http://www.bundeswahlleiter.de/de/bundestagswahlen/BTW_BUND_13/vero effentlichungen/BTW2013_1_Bund.pdf (Stand: 23.12.2013).

Bündnis 90/Die Grünen. Zeit für den grünen Wandel. Bundestagswahlprogramm 2013. Berlin: Bündnis 90/Die Grünen, http://www.gruene.de/fileadmin/user_upload/ Dokumente/Wahlprogramm/Wahlprogramm-barrierefrei.pdf (Stand: 23.12. 2013).

Butzlaff, Felix. "Gewonnen und verloren - sozialdemokratische Stimmenarithmetik." 26. September 2013, http://www.demokratie-goettingen.de/blog/gewonnen -und-verloren (Stand: 23.12.2013). 
Dostal, Jörg Michael. "Explaining the Crisis and Electoral Decline of the German Social Democratic Party (SPD) in the Era of Welfare State Retrenchment." Zeitschrift der Koreanisch-Deutschen Gesellschaft für Sozialwissenschaften. 22. Jg., Heft 4, 2012, S. 271-310.

Europäische Kommission. "Anhang 2. Trends bei Leistungsbilanzen und Lohnstückkosten," 11. März 2013, http://europa.eu/rapid/press-release_ MEMO-13-198_de.htm (Stand: 23.12.2013).

European Commission. "Standard Eurobarometer 79, Spring 2013, Tables of Results," http://ec.europa.eu/public_opinion/archives/eb/eb79/eb79_anx_en.pdf (Stand 23.12.2013).

FAZ. "Peer Steinbrück im Gespräch: Bundeskanzler verdient zu wenig." Frankfurter Allgemeine Zeitung, 29.12.2012.

GfK-Verein. "Challenges of Europe 2013." Powerpoint Presentation. Nürnberg: GfK-Verein, Juni 2013.

Heidar, Knut und Jo Saglie. "Predestined Parties? Organizational Change in Norwegian Political Parties." Party Politics. 9. Jg., Heft 2, 2003, S. 219-239.

Huntington, Samuel P. "The United States." Crozier, M. J., S. P. Huntington und J. Watanuki (Hrsg.). The Crisis of Democracy: Report on the Governability of Democracies to the Trilateral Commission. New York: New York University Press, 1975, S. 59-118.

Infratest Dimap a.ARD-DeutschlandTREND, September 2013, http://www.infratestdimap.de/uploads/media/dt1309_bericht_01.pdf (Stand: 23.12.2013).

Infratest Dimap b. "Wähler nach Altersgruppe" und "Wähler nach Tätigkeit," Spiegel online, 22. September 2013, http://www.spiegel.de/politik/deutschland/ bundestagswahl-2013-waehlerwanderung-gewinne-verluste-direktman date-a-923290.html (Stand: 23.12.2013).

Jun, Uwe. "Volksparteien under Pressure: Challenges and Adaptation." German Politics. 20. Jg., Heft 1, S. 200-222. 
Die Bundestagswahl 2013: Demokratische Beteiligungslücke und Dauerkrise der SPD 115

Katz, Richard S. und Peter Mair. "Changing Models of Party Organization and Party Democracy: The Emergence of the Cartel Party." Party Politics. 1. Jg., Heft 1, 1995, S. 5-28.

Kirchheimer, Otto. "Der Wandel des westeuropäischen Parteiensystems." Politische Vierteljahresschrift. 6. Jg., Heft 1, 1965, S. 20-41.

Kirchheimer, Otto. "The Transformation of Western European Party Systems." La Palombara, Joseph und Myron Weiner (Hrsg.). Political Parties and Political Development. Princeton, NJ: Princeton University Press, 1966, S. 177-200. Korte, Karl-Rudolf. "Die Bundestagswahl 2009 -Konturen des Neuen. Problemstellungen der Regierungs-, Parteien-, Wahl- und Kommunikationsforschung." Korte, Karl-Rudolf (Hrsg.). Die Bundestagswahl 2009. Analysen der Wahl-, Parteien-, Kommunikations - und Regierungsforschung. Wiesbaden: VS Verlag, 2010, S. 9-32.

Kommission Grundwerte. "Die Zukunft der SPD als Volkspartei." Kommission Grundwerte beim Parteivorstand der SPD, 2010, https://www.spd.de/spdwebapp/servlet/elementblob/10525599/content (Stand: 23.12.2013).

Koole, Ruud. "The Vulnerability of the Modern Cadre Party in the Netherlands." Katz, Richard und Peter Mair (Hrsg.). How Parties Organize. London: Sage, 1994, S. 278-303.

Kulick, Manuela S. "Absturz der Volksparteien. Eine Analyse der loyalen und ehemaligen Wählerschaft von CDU und SPD im Vergleich." Niedermayer, Oskar (Hrsg.). Die Parteien nach der Bundestagswahl 2009. Wiesbaden: Verlag für Sozialwissenschaften, 2011, S. 199-219.

Lijphart, Arend. "Unequal Participation: Democracy's Unresolved Dilemma." American Political Science Review. 91. Jg., Heft 1, 1997, S. 1-14.

Linden, Markus und Winfried Thaa. "Krise und Repräsentation." Linden, Markus und Winfried Thaa (Hrsg.). Krise und Reform politischer Repräsentation. Baden Baden: Nomos, 2011, S. 11-41.

Lühmann, Michael. "Der Fehler der Grünen: Zu viel Trittin, zu wenig KGE." Cicero-online, 12. September 2013, http://www.cicero.de/berliner-republik 
/gruener-sinkflug-der-fehler-der-gruenen-zu-viel-trittin-zu-wenig -katrin-goering-eckardt/55739?print (Stand: 23.12.2013).

Merkel, Wolfgang. "Der Parteiverächter. Wider den publizistischen Stammtisch - ein Zwischenruf." WZB-Mitteilungen. Heft 124, 2009, S. 12-16.

Nachtwey, Oliver. "Market Social Democracy: The Transformation of the SPD up to 2007." German Politics. 22. Jg., Heft 3, 2013, S. 235-252.

Nawrat, Sebastian. Agenda 2010 - ein Überraschungscoup? Kontinuität und Wandel in den wirtschafts - und sozialpolitischen Programmdebatten der SPD seit 1982. Bonn: J. H. W. Dietz, 2012.

Neu, Viola. "Bundestagswahl in Deutschland am 22. September 2013. Wahlanalyse." Berlin: Konrad Adenauer Stiftung, Hauptabteilung Politik und Beratung, September 2013.

Neugebauer, Gero. "Die Unterschicht und die Parteien." Aus Politik und Zeitgeschichte 33-34/2008. Bonn: Bundeszentrale für politische Bildung, 2008, S. 31-38. Niedermayer, Oskar. "Der Wandel des parteipolitischen Engagements der Bürger." Kühnel, Steffen, Oskar Niedermayer und Bettina Westle (Hrsg.). Wähler in Deutschland. Sozialer und politischer Wandel, Gender und Wahlverhalten. Wiesbaden: VS Verlag, 2009, S. 82-134.

Niedermayer, Oskar. "Triumph und Desaster: Die SPD im deutschen Parteiensystem nach der Vereinigung." Gesellschaft, Wirtschaft, Politik (GWP). 59. Jg., Heft 2, 2010, S. 225-236.

Niedermayer, Oskar. "Parteimitgliedschaften im Jahre 2012." Zeitschrift für Parlamentsfragen. 44. Jg., Heft 2, 2013, S. 365-383.

Nijhuis, Tom. "Regieren und Parteienwettbewerb in einem nivellierten Vielparteiensystem." Korte, Karl-Rudolf (Hrsg.). Die Bundestagswahl 2009. Analysen der Wahl-, Parteien-, Kommunikations - und Regierungsforschung. Wiesbaden: VS Verlag, 2010, S. 130-148.

Panebianco, Angelo. Political Parties: Organization and Power. Cambridge (UK): Cambridge University Press, 1988. 
Die Bundestagswahl 2013: Demokratische Beteiligungslücke und Dauerkrise der SPD 117

Pharr, Susan J., Robert D. Putnam und Russel J. Dalton. "A Quarter-Century of Declining Confidence." Diamond, Larry und Marc F. Plattner (Hrsg.). The Global Divergence of Democracies. Baltimore: Johns Hopkins University Press, 2001, S. 291-311.

Schäfer, Armin. "Der Nichtwähler als Durchschnittsbürger: Ist die sinkende Wahlbeteiligung eine Gefahr für die Demokratie?" Bytzek, Evelyn und Sigrid Roßteutscher (Hrsg.). Der unbekannte Wähler? Mythen und Fakten über das Wahlverhalten der Deutschen. Frankfurt/M.: Campus Verlag, 2011, S. $133-154$.

Schäfer, Armin. "Beeinflusst die sinkende Wahlbeteiligung das Wahlergebnis? Eine Analyse kleinräumiger Wahldaten in deutschen Großstädten." Politische Vierteljahresschrift. 53. Jg., Heft 2, 2012, S. 240-264.

Schäfer, Armin. "Liberalization, Inequality and Democracy's Discontent." Schäfer, Armin und Wolfgang Streeck (Hrsg.). Politics in the Age of Austerity. Cambridge (UK): Polity Press, 2013, S. 169-195.

Sinus. "Informationen zu den Sinus-Milieus 2011." Heidelberg: Sinus Markt und Sozialforschung, 04/2011.

Sirleschtov, Antje. "Schwarz-Gelb im Streit über Zweitstimmen." Cicero-online, 19. September 2013, http://www.cicero.de/berliner-republik/bundestagswahlkampf-um-zweitstimmen-entzweit-schwarz-gelb/55828 (Stand: 23.12. 2013).

Stöss, Richard und Gero Neugebauer. "Die SPD und die Bundestagswahl 1998." Arbeitshefte aus dem Otto-Stammer-Zentrum Nr. 2. Berlin: Freie Universität Berlin, 1998.

Streeck, Wolfgang. Re-forming Capitalism. Institutional Change in the German Political Economy. Oxford: Oxford University Press, 2009.

Streeck, Wolfgang und Armin Schäfer. "Introduction: Politics in the Age of Austerity." Schäfer, Armin und Wolfgang Streeck (Hrsg.). Politics in the Age of Austerity. Cambridge (UK): Polity Press, 2013, S. 1-25. 
Tagesschau. "Analysen Wählerwanderung." 27.9.2009, http://wahl.tagesschau.de/ wahlen/2009-09-27-BT-DE/analyse-wanderung.shtml （Stand：23.12. 2013).

Tagesschau. "Analysen Wählerwanderung." 22.9.2013, http://wahl.tagesschau.de/ wahlen/2013-09-22-BT-DE/analyse-wanderung.shtml （Stand）23.12. 2013).

Verba, Sidney, Kay Lehman Schlozman und Henry E. Brady. "Political Equality: What Do We Know About It?" Neckerman, Kathryn M. (Hrsg.). Social Inequality. New York: Russel Sage Foundation, 2004, S. 635-666.

Walter, Franz. "Identitätskrise der SPD: Ratlose Bluthunde." Tageszeitung (taz), 24.6.2013.

Walter, Franz, Christian Werwath und Oliver D'Antonio. Die CDU. Entstehung und Verfall christdemokratischer Geschlossenheit. Baden Baden: Nomos, 2011. Wiesendahl, Elmar. "Partizipation in Parteien: Ein Auslaufmodell?" Hoecker, Beate (Hrsg.). Politische Partizipation zwischen Konvention und Protest. Opladen: Verlag Barbara Budrich, 2006, S. 74-99.

Zolleis, Udo und Carina Wertheimer. "Is the CSU Still a Volkspartee?" German Politics. 22. Jg., Heft 1, 2013, S. 97-113.

Zolo, Danilo. Democracy and Complexity. A Realist Approach. Übers. von David McKie. Pennsylvania: Pennsylvania State University Press, 1992. 
Die Bundestagswahl 2013: Demokratische Beteiligungslücke und Dauerkrise

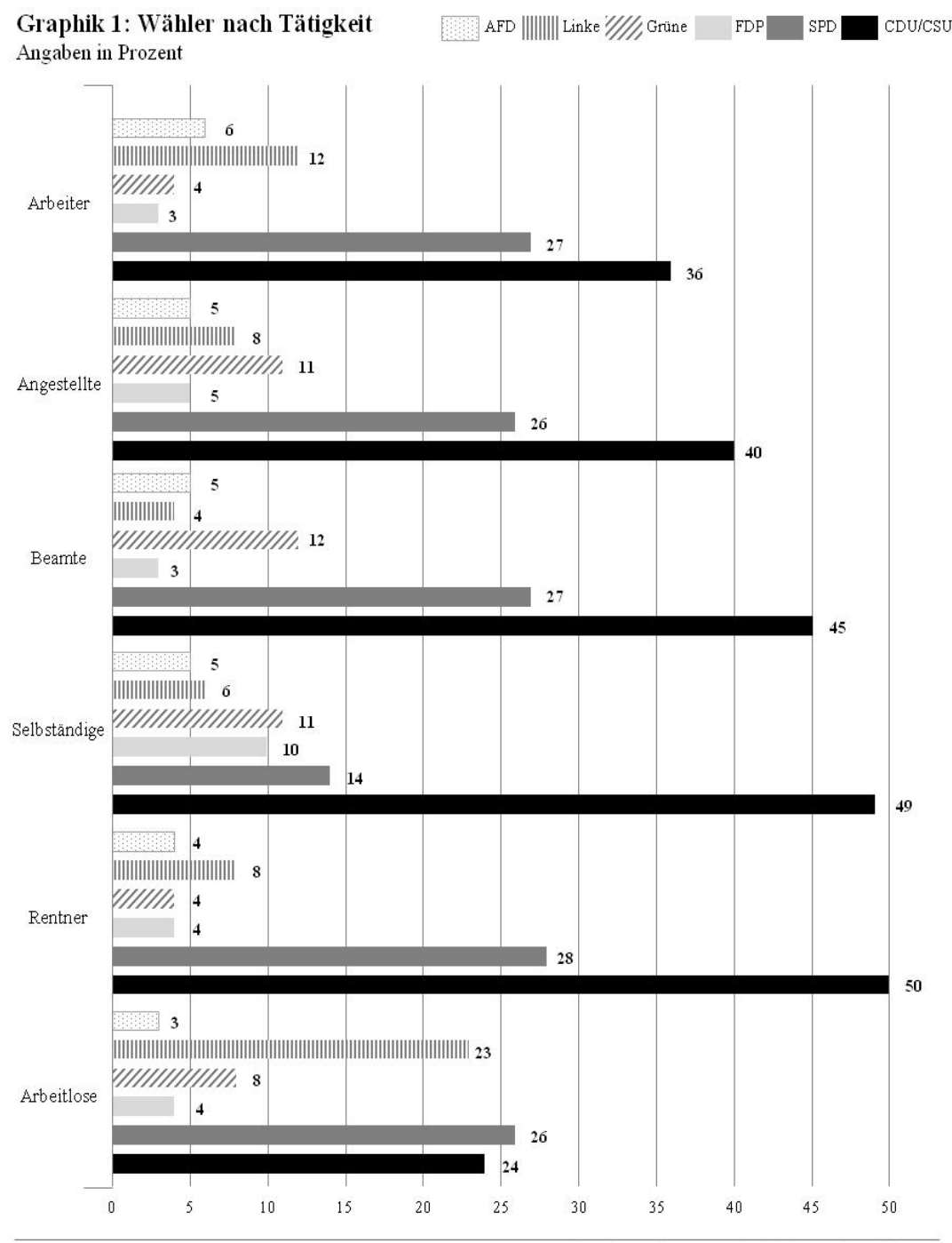

Quelle: Infratest dimap, Stand: Vorläufiges Endergebnis 
120 한 - 독사회과학논총 2013년 겨울

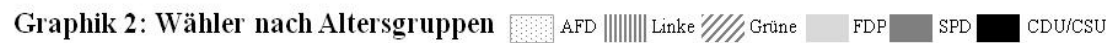
Angaben in Prozent

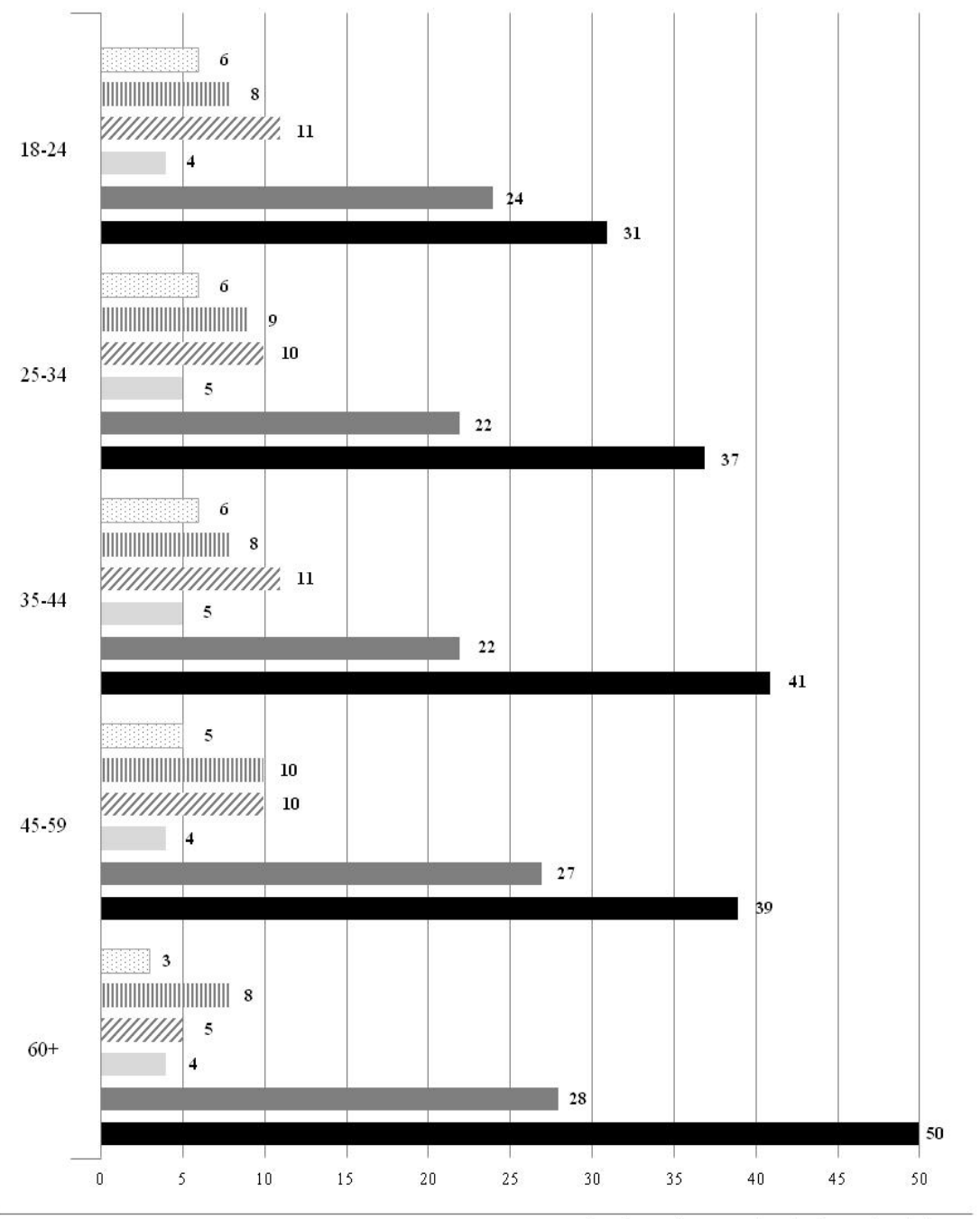

Quelle: Infratest dimap, Stand: Vorläufiges Endergebnis 
Die Bundestagswahl 2013: Demokratische Beteiligungslücke und Dauerkrise

Tabelle 1: Zweitstimmenanteil und Wählerwanderung der Parteien, Bundestagswahl 2013

\begin{tabular}{|c|c|c|c|c|c|c|c|c|}
\hline \multicolumn{2}{|c|}{} & CDU/CSU & SPD & DIE LINKE & GRÜNE & FDP & AfD \\
\hline CDU/CSU & $41.5 \%$ & $(+7.7 \%)$ & - & $+210,000$ & $+120,000$ & $+420,000$ & $+2,110,000$ & $-290,000$ \\
\hline SPD & $25.7 \%$ & $(+2.7 \%)$ & $-210,000$ & - & $+370,000$ & $+550,000$ & $+530,000$ & $-180,000$ \\
\hline DIE LINKE & $8.6 \%$ & $(-3.3 \%)$ & $-120,000$ & $-370,000$ & - & $-40,000$ & $+90,000$ & $-340,000$ \\
\hline GRÜNE & $8.4 \%$ & $(-2.3 \%)$ & $-420,000$ & $-550,000$ & $+40,000$ & - & $+170,000$ & $-90,000$ \\
\hline FDP & $4.8 \%$ & $(-9.6 \%)$ & $-2,110,000$ & $-530,000$ & $-90,000$ & $-170,000$ & - & $-430,000$ \\
\hline AfD & $4.7 \%$ & $(+4.7 \%)$ & $+290,000$ & $+180,000$ & $+340,000$ & $+90,000$ & $+430,000$ & - \\
\hline
\end{tabular}

Lesebeispiel: Die Tabelle zeiggt die Nettostimmenwanderung, so gewann die CDU/CSU z.B. 210,000

Quelle:Tag̣esschau, 22.9.2013

Stimmen von der SPD und verlor 290,000 Stimmen an die AfD.

Tabelle 2: Mobilisierungspotential(Anzahl der Zweitstimmen) der im Bundestag vertretenen Parteien (1990-2013)

\begin{tabular}{|c|c|c|c|c|c|c|c|c|c|c|}
\hline & \multicolumn{2}{|c|}{$\mathrm{CDU} / \mathrm{CSU}$} & \multicolumn{2}{|l|}{ SPD } & \multicolumn{2}{|c|}{ DIE LINKE* } & \multicolumn{2}{|c|}{ GRÜNE } & \multicolumn{2}{|c|}{ FDP } \\
\hline 1990 & $20,358,096$ & $100.0 \%$ & $15,545,366$ & $77.0 \%$ & $1,129,578$ & $21.9 \%$ & $2,347,407$ & $50.6 \%$ & $5,123,233$ & $81.1 \%$ \\
\hline 1994 & $19,517,156$ & $95.9 \%$ & $17,140,354$ & $84.9 \%$ & $2,066,176$ & $40.1 \%$ & $3,424,315$ & $73.7 \%$ & $3,258,407$ & $51.6 \%$ \\
\hline 1998 & $17,329,388$ & $85.1 \%$ & $20,181,269$ & $100.0 \%$ & $2,515,454$ & $48.8 \%$ & $3,301,624$ & $71.1 \%$ & $3,080,955$ & $48.8 \%$ \\
\hline 2002 & $18,482,641$ & $90.8 \%$ & $18,488,668$ & $91.6 \%$ & $1,916,702$ & $37.2 \%$ & $4,110,355$ & $88.5 \%$ & $3,538,815$ & $56.0 \%$ \\
\hline 2005 & $16,631,049$ & $81.7 \%$ & $16,194,665$ & $80.2 \%$ & $4,118,194$ & $79.9 \%$ & $3,838,326$ & $82.7 \%$ & $4,648,144$ & $73.6 \%$ \\
\hline 2009 & $14,658,515$ & $72.0 \%$ & $9,990,488$ & $49.5 \%$ & $5,155,933$ & $100.0 \%$ & $4,643,272$ & $100.0 \%$ & $6,316,080$ & $100.0 \%$ \\
\hline 2013 & $18,165,446$ & $89.2 \%$ & $11,252,215$ & $55.8 \%$ & $3,755,699$ & $72.8 \%$ & $3,694,057$ & $79.6 \%$ & $2,083,533$ & $33.0 \%$ \\
\hline
\end{tabular}

* Bis 2005 Partei des demokratischen Sozialismus (PDS)

Quelle: Bundeswahlleiter, 2013b und eigene Berechnungen 


\section{3 독일연방의회선거: 민주적 참여공백과 사민당의 지속적 위기}

요륵 미샤엘 도스탈(Jörg Michael Dostal)*

\section{<국문초록>}

2013년 독일연방선거에서 기민당/기사당(CDU/CSU)의 옛 연정파트너였던 자유민 주당(FDP)의 패배에 힘입어 기민당/기사당(CDU/CSU)이 비교적 높은 지지율을 획득 하였음에도 불구하고, 독일의 정당시스템은 두 가지 측면의 구조적 위기에 직면하고 있다. 첫째, 투표참여가 크게 줄어든 것으로서, 전통적인 양대 국민정당 (기민당/기사 당 $(\mathrm{CDU} / \mathrm{CSU})$, 사민당 $(\mathrm{SPD})$ ) 지지층의 정당에 대한 결속력이 급격히 줄어들고 있는 것을 들 수 있으며, 이에 따라 양대 국민정당들은 와해의 국면에 처해있다. 둘째, 비대 칭적인 위기에 관한 것으로서, 기민당/기사당(CDU/CSU)은 그나마 비교적 완만한 속 도로 지지율을 잃고 있는 반면, 사민당(SPD)의 정당지지율은 급속히 감소하고 있으며, 당원 또한 크게 줄어들고 있는 상황이다. 이러한 변화에는 옛 동독지역과 서독지역간 의 사회적 양극화 증가가 가장 큰 원인으로 작용하고 있다. 즉, 일반시민계층 지지자들 의 투표권 행사 비율은 비교적 높게 유지되고 있는 것으로 나타나고 있지만, 여러가지 다른 원인들과 함께 사회적 소외계층들의 투표참여가 줄어들고 있는 사회적 양극화 증 가가 가장 큰 원인으로 작용하고 있다. 기존의 일반시민계층에 기반을 둔 정당들과 비 교하여 사민당(SPD)과 좌파당(Die Linke)은 사회적 약자들의 지지 기반이 더 절실한 실정임에도, 민주적 참여공백이 나타나고 있어 특히 사민당(SPD)과 옛 동독지역의 좌 파당(Die Linke)이 영향을 받고 있다.

주제어: 2013 독일연방의회선거, 독일, 투표참여감소, 독일사회민주당(SPD), 국민정당의 위기

논문접수일자: 2013년 11월 06일,논문심사일자: 2013년 11월 24일 게재확정일자: 2013년 12월 22일

* 서울대학교 행정대학원 교수 